\title{
Identification of a novel modulator of the actin cytoskeleton, mitochondria, nutrient metabolism and lifespan in yeast
}

Cierra N. Sing ${ }^{1,2}$, Enrique J. Garcia ${ }^{1}$, Thomas G. Lipkin ${ }^{1,3}$, Thomas M. Huckaba ${ }^{1,4}$, Catherine A. Tsang $^{1}$, Arielle C. Coughlin ${ }^{1}$, Emily J. Yang ${ }^{1}$, Istvan R. Boldogh ${ }^{1}$, Ryo Higuchi-Sanabria ${ }^{1,2,5}$ and Liza A. Pon ${ }^{1,2^{*}}$

${ }^{1}$ Department of Pathology and Cell Biology, Columbia University, New York, NY 10032

${ }^{2}$ Institute of Human Nutrition, Columbia University, New York, NY 10032

${ }^{3}$ Technology Development Group, University of California, Los Angeles, CA 90095

${ }^{4}$ Department of Biology, Xavier University of Louisiana, New Orleans, LA 70125

${ }^{5}$ Leonard Davis School of Gerontology, University of Southern California, Los Angeles, CA 90089

${ }^{*}$ Please address correspondence to:

Liza A. Pon, Ph.D.

Department of Pathology and Cell Biology

Columbia University

630 W. $168^{\text {th }}$ St. P\&S $14-442$

New York, NY 10032

Tel: (212) 305-1947

e-mail: lap5@cumc.columbia.edu 


\section{Abstract}

In yeast, actin cables are F-actin bundles that are essential for cell division through their function as tracks for cargo movement from mother to daughter cell. Actin cables also affect yeast lifespan by promoting transport and inheritance of higher-functioning mitochondria to daughter cells. Here, we report that actin cable stability declines with age. Our genome-wide screen for genes that affect actin cable stability identified the open reading frame YKLO75C. Deletion of $Y K L 075 C$ results in increases in actin cable stability and abundance, mitochondrial fitness, and replicative lifespan. Transcriptome analysis revealed a role for $Y K L 075 C$ in regulating branched-chain amino acid (BCAA) metabolism. Consistent with this, modulation of BCAA metabolism or decreasing leucine levels promotes actin cable stability and function in mitochondrial quality control. Our studies support a role for actin stability in yeast lifespan, and demonstrate that this process is controlled by BCAA and a previously uncharacterized ORF YKL075C, which we refer to as actin, aging and nutrient modulator protein 1 (AAN1). 


\section{Introduction}

The actin cytoskeleton is critical, not just for normal cellular function, but for lifespan and healthspan, the quality of life during the aging process. For example, actin function declines with age in the immune system, which contributes to defects in 1) phagocytosis by macrophages, 2) exocytosis in neutrophils, 3) formation of the immunological synapse between T cells and antigen-presenting cells, and 4) migration of mesenchymal stem cells to sites of injury ${ }^{1-11}$. Similarly, the speed of actin sliding on skeletal muscle myosin and maximum velocity of muscle fiber shortening decline during the aging process, which may contribute to age-linked loss of muscle function $^{12-16}$.

The actin cytoskeleton is also critical for mother-daughter age asymmetry, the process whereby babies are born young, largely independent of the age of their parents. This process is conserved from bacteria to humans and is driven at the single-cell level by the asymmetric inheritance of aging determinants, which preserve mother cell age and rejuvenate daughter cells. Oxidatively damaged, carbonylated proteins and protein aggregates are retained exclusively in yeast mother cells and defects in this process reduce lifespan and abolish motherdaughter age asymmetry ${ }^{17,18}$. Conversely, higher-functioning populations of organelles including mitochondria and the yeast lysosome (vacuole) are preferentially inherited by yeast daughter cells $^{19-22}$. Interestingly, mitochondria are also asymmetrically inherited during cell division in human mammary stem-like cells, and this process affects cell fate ${ }^{23}$.

The asymmetric inheritance of aging determinants in yeast is dependent on actin cables, tracks for polarized movement of virtually all cargoes in yeast. Actin cables assemble in the bud, extend along the mother-bud axis and disassemble in the mother cell tip ${ }^{24-29}$. However, in contrast to most cytoskeletal tracks, which are stationary, our previous studies revealed that these structures are dynamic and undergo retrograde flow, treadmill-like movement away from the bud and towards the mother cell ${ }^{24}$. As a result, actin cables act as filters to prevent the 
transport and inheritance of lower-functioning mitochondria from mother to daughter cells. Indeed, we found that promoting actin cable dynamics promotes mitochondrial function and extends lifespan in yeast ${ }^{20}$.

Although cytoskeletal function is critical for lifespan control in yeast and other eukaryotes, how actin changes with age, and the mechanisms underlying this process, are not well understood. Here, we obtained evidence that the stability of actin cables declines with age in budding yeast. We also identified a role for a previously uncharacterized gene (YKLO75C) in regulating actin cable stability, mitochondrial quality, branched-chain amino acid (BCAA) metabolism and longevity.

\section{Results}

\section{Actin cable integrity declines with age in yeast}

Here, we visualized actin cables as a function of age in yeast undergoing replicative aging, a model of lifespan control that is based on the number of times a yeast mother cell divides before senescence ${ }^{30}$. Briefly, mid-log phase yeast cells, which are primarily young cells, were labeled on their cell walls with biotin. These cells were then propagated in shake flasks or in a mini-chemostat aging device (mCAD), which resulted in production of young, unlabeled cells by budding from the original biotinylated population ${ }^{31,32}$. Older, biotinylated cells were then separated from young, unlabeled cells using streptavidin affinity purification ${ }^{31,32}$. The replicative age of isolated young and old cells was determined by assessing the number of bud scars, ringshaped structures on the cell wall of mother cells that form at the site of mother-daughter separation (Fig. 1a).

We visualized the actin cytoskeleton of young and old yeast cells by staining with fluorochrome-coupled phalloidin and imaging by super-resolution structured illumination microscopy (SIM). We confirmed that young cells contain the 2 actin-containing structures that 
are present throughout the cell cycle: actin patches (endosomes that have an F-actin coat and are enriched in the bud) and actin cables (bundles of F-actin that extend along the mother-bud axis and function in cargo transport) (Fig.1a). Moreover, we observed morphological and organizational changes in the actin cytoskeleton in old yeast cells (> 10 generations in replicative age). Specifically, we found that F-actin content in actin cables, assessed by the intensity of staining using fluorochrome-coupled phalloidin, decreases with age (Fig. 1a,b). Consistent with this, we found that the apparent width of actin cables declines with age (Extended data Fig. 1a). Finally, in young cells, all actin cables are polarized along the motherbud axis. In contrast, depolarized actin cables are evident in cells of advanced age (> 10 generations) (Fig. 1a).

To determine whether the observed cytoskeletal changes are due to effects on actin stability, we assessed the sensitivity of actin cables of young and old cells to destabilization by Latrunculin A (Lat-A). Lat-A promotes loss of F-actin structures by two mechanisms. It inhibits actin polymerization by sequestering monomeric actin, and stimulates actin depolymerization by promoting release of $\mathrm{Pi}$ from actin bound to ADP and $\mathrm{Pi}$ within actin polymers ${ }^{33}$.

Here, we used concentrations of Lat-A that preferentially affect actin cables. Previous studies indicate that high levels of Lat-A $(200 \mu \mathrm{M})$ induces a loss of all F-actin-containing structures in yeast, while exposure to low Lat-A concentrations (1-10 $\mu \mathrm{M})$ results in actin cable loss with no effects on actin patches (Extended Data Fig. 1b) ${ }^{34}$. We isolated young and old yeast using the mCAD as described above and monitored actin cable content in these cells as a function of the time of treatment with low levels of Lat-A. As expected, we observed a loss of actin cables with Lat-A treatment in young cells. In addition, we detected a statistically significant increase in the rate of Lat-A-induced actin cable loss in old compared to young yeast cells (Fig. 1c,d). Thus, actin cable stability declines with age in yeast.

\section{Identification of genes that are critical for actin cable stability}


Since actin cables are essential for transport of cellular constituents to buds during bud formation and growth, Lat-A-induced loss of actin cables inhibits yeast cell growth (Fig. 1e). Therefore, to identify genes that are critical for actin cable stability, we screened a yeast strain collection that bears a deletion in each of the non-essential yeast genes for deletions that result in reduced sensitivity to Lat-A-induced growth inhibition (Fig. 1e). 18 hits were identified in this screen. None of the deletions identified had effects on growth rates in the absence of Lat-A (Extended Data Fig. 1c). However, in the presence of low levels of Lat-A, all 18 deletion strains exhibited increased growth rates compared to wild-type cells (Fig. 1e, Extended Data Fig. 1d). 13 of the 18 strains also exhibited a significant increase in actin cable abundance compared to wild-type cells (Fig. 1f). Two of the hits that exhibit normal actin cable abundance in the absence of Lat-A treatment, BRO1 and FAR10, are genes that function in membrane transport. These hits, and other hits that produce the same phenotype, may impact on Lat-A sensitivity through effects on uptake or export of the drug.

To determine whether the deletions affect overall cell polarity, we studied the localization of actin patches. These structures are enriched in the bud, which reflects endocytosis at that site, presumably to promote recycling of membrane components at sites of membrane deposition during bud growth. We do not detect depolarization of actin patches in any of the hits studied. Thus, using an unbiased approach, we identified genes that, when deleted, increase actin cable abundance and reduce yeast cell sensitivity to destabilization of actin cables by LatA treatment.

Two of the hits identified in the screen encode previously uncharacterized open reading frames, YHR022C and YKL075C. Moreover, none of the hits were in genes that encode known actin binding or regulatory proteins. Therefore, we carried out SGD GO SLIM analysis of the 17 characterized gene hits. This analysis revealed a role for 2 or more of the hits in processes including response to chemical stressors, cell cycle, DNA recombination, regulation or repair, 
amino acid metabolism, translation, protein targeting and endosomal transport (Extended Fig. 1e).

\section{YKL075C affects actin cable stability and replicative lifespan}

Our efforts focused on YKLO75C, one of the 2 uncharacterized genes identified in our screen. Since strains in the yeast deletion collection can carry secondary mutations with functional consequences ${ }^{35}$, we constructed new YKL075C deletion strains. In newly constructed strains, we confirmed that deletion of $Y K L 075 C$ 1) does not affect yeast cell growth or polarity (Fig. 2a,b), 2) reduces the sensitivity of yeast to the growth-inhibiting effects of low-level Lat-A treatment (Fig. 2a), and 3) increases actin cable abundance (Fig. 2b,c). Since another open reading frame (YKLO76C) overlaps with the C-terminal coding region of YKL075C, it is possible that the phenotypes observed in $y$ k1075c $\Delta$ cells are due to loss of the overlapping gene. However, we find that expression of the YKL075C gene under control of its endogenous promoter in $y k 1075 c \Delta$ cells reduced the actin cable abundance to wild-type levels (Extended Fig. 2a,b). Thus, we found that ectopic expression of YKL075C complements loss of the gene. Together, our findings support a role for $Y K L 075 \mathrm{C}$ in modulating actin cable abundance in budding yeast.

Next, we tested whether deletion of YKL075C promotes actin cable stability using the Lat-A sensitivity assay described above. We found that actin cables in $y k 1075 c \Delta$ cells exhibit reduced sensitivity to Lat-A treatment compared to wild-type yeast (Fig. 2d,e). We also found that deletion of $Y K L O 75 C$ has no effect on the rate of retrograde actin cable flow (Extended Data Fig. 2c,d). Thus, we identified a novel modulator that affects actin cable stability and abundance but has no detectable effect on actin cable treadmilling.

Since actin cable stability declines with age, we tested whether stabilization of actin cables by deletion of YKL075C promotes yeast cell lifespan. Here, we studied the replicative lifespan (RLS) of yeast, the number of times that a mother cell divides before senescence. We 
found that deletion of YKLO75C extends lifespan in yeast. Specifically, the mean RLS of $y k 1075 c \Delta$ cells (26 generations) is significantly greater than that of wild-type cells (19 generations) (Fig. 2f). Although reduced RLS can result from nonspecific factors, interventions that extend RLS must affect processes that limit lifespan. Thus, our finding that deletion of YKL075C extends RLS supports a role for that gene in the aging process in yeast.

Finally, we assessed the role of $Y K L 075 C$ in cellular healthspan by examining 2 hallmarks of cellular fitness: 1) mitochondrial function and 2) mean generation time (the time required for a cell to complete a round of cell division). Mitochondrial function was evaluated using a ratiometric sensor for the redox state of the mitochondrial matrix (mito-roGFP1) ${ }^{19,36,37}$. We found that mitochondria are more reduced, and therefore higher functioning, in $y k 1075 c \Delta$ cells compared to wild-type cells (Fig. 2g,h). Equally important, we found deletion of YKL075C does not affect asymmetric inheritance of mitochondria in yeast: fitter mitochondria, that are more reduced, are preferentially inherited by yeast daughter cells in both $y k 1075 c \Delta$ and wild-type cells (Extended Data Fig. 2e). Thus, deletion of YKL075C promotes the function of mitochondria, an aging determinant that is asymmetrically inherited in yeast.

Consistent with this, we found that the mean generation time (MGT) is reduced in $y k 1075 c \Delta$ cells (Extended Data Fig. 2f). We confirmed previous findings that the MGT of wildtype yeast cells increases with age. We also found that the MGT of young (0-10 generation) wild-type and $y k 1075 c \Delta$ cells is similar. This finding provides additional evidence that deletion of YKL075C does not affect the growth rate of mid-log phase yeast, which are primarily young cells. While the MGT of $y k 1075 c \Delta$ cells increased with age, the rate of increase of MGT with age in $y k 1075 c \Delta$ cells is significantly less than that of wild-type cells. Thus, we identified a novel role for the previously uncharacterized protein, Ykl075cp, in modulating actin cable stability and abundance, lifespan and 2 markers of healthspan, mitochondrial function and generation time.

\section{YKL075C targets branched-chain amino acid homeostasis}


YKL075C has no obvious functional domains or sequence similarity to known proteins. Since YKL075C affects actin cables, we tested whether the protein encoded by the YKL075C gene (Ykl075cp) co-localizes with the actin cytoskeleton in yeast. We tagged the endogenous YKL075C gene at its C terminus with GFP and found that the signal obtained by fluorescence imaging was weak. This finding is consistent with reports that Ykl075cp is not an abundant protein $^{38}$. Therefore, we tagged the gene with 13 copies of the Myc epitope. We found that tagging YKL075C at its chromosomal locus results in expression of a protein of the expected apparent molecular weight (Extended Data Fig. 3a), and does not disrupt the cell growth rate. Consistent this, we found that expression of Myc-tagged YKLO75C complements loss of the gene and restores actin cable abundance to wild-type levels (Extended Data Fig. 3b,c). Since tagging YKL075C with 13-Myc does not affect its function, we studied the localization of the tagged protein and actin cytoskeleton in mid-log phase yeast (Fig. 3a). Ykl075cp localizes to punctate cytosolic structures in mother cells and buds. There is no obvious co-localization of Ykl075cp with actin patches or cables. Thus, YLK075C effects on actin cables is not through direct effects on those structures or on endosomes.

Next, we used RNA-Seq to study gene expression in yeast bearing a deletion in YKL075C (Supplementary Table 1). This analysis revealed transcriptional changes in processes ranging from amino acid metabolism and the mitochondrial electron transport chain to iron homeostasis and transport (Fig. 3b). Among these, there were significant decreases in levels of transcripts encoding branched-chain amino acid (BCAA) biosynthetic processes in $y k 1075 c \Delta$ compared to wild-type cells (Fig. 3b,c). Notably, BAT1, which encodes the BCAA transaminase (BAT) that catalyzes the last step of BCAA synthesis ${ }^{39}$, and 5 out of 10 other BCAA biosynthetic genes are down-regulated in $y k / 075 c \Delta$ cells. In addition, there is an increase in expression of BAT2, a BAT1 paralog that preferentially mediates BCAA catabolism ${ }^{40,41}$.

Interestingly, GO term analysis revealed that other hits identified the Lat-A screen function in amino acid metabolism or endosomal transport, a process that impacts on the amino 
transport and on the organelle (the vacuole) that stores and contributes to amino acid sensing. These findings support a role for amino acids in modulation of the actin cytoskeleton and raise the possibility that other hits identified in the screen may function with Aan $1 p$ in cytoskeletal control pathways.

To validate the transcriptome studies, we assessed the levels of specific BCAA biosynthetic genes and of BCAAs. Using qPCR, we observed a $65 \%$ reduction in BAT1 expression, and a 2-fold increase in BAT2 expression in yk1075c $\Delta$ cells compared to wild-type cells (Fig. 3d,e). We also detected a significant reduction in the level of free BCAAs in whole-cell extracts from $y k 1075 c \Delta$, bat $1 \Delta$, and $y k / 075 c \Delta$ bat $1 \Delta$ cells compared to wild-type cells (Fig. 3f). Importantly, while we observed a decrease in BCAA levels in both single and double deletion strains compared to wild-type cells, we found no additive effect of the simultaneous deletion of YKLO75C and BAT1 compared to bat1 $\triangle$ single mutants. These findings indicate that 1) YKL075C, like BAT1, modulates BCAA levels, and 2) YKL075C and BAT1 function in the same pathway for BCAA control. Collectively, our findings indicate that $Y K L 075 \mathrm{C}$ affects actin cables, key nutrients, and lifespan in yeast. Therefore, we will refer to YKL075C as AAN1: a actin, aging and nutrient modulator protein 1.

TORC1 (Target of rapamycin complex 1) regulates anabolic cellular processes and lifespan in response to nutrients including BCAAs ${ }^{42-44}$. Therefore, TORC1 may contribute to Aan $1 \mathrm{p}$ function in modulating actin cables and lifespan. To test this hypothesis, we tested whether TORC1 is activated by deletion of AAN1. TOR1 and TOR2, the 2 TOR paralog genes of yeast, are subunits of TORC $1^{42,45}$. Since TOR2 is an essential gene, TOR1 nulls were used as control to evaluate TORC1 activity ${ }^{46}$. We confirmed previous findings that deletion of TOR1 delays re-entry into the cell cycle after release from rapamycin-induced inhibition of cell growth $^{47,48}$. Specifically, we found that the growth of tor $1 \Delta$ cells is reduced compared to wildtype cells after removal of rapamycin from the growth medium (Fig. 3g,h). On the other hand, 
the recovery of aan $1 \Delta$ cells from rapamycin treatment is indistinguishable from that of wild-type cells and distinct from that observed in tor1 $\Delta$ or aan1 $1 \Delta$ tor1 $1 \Delta$ mutants (Fig. 3g,h).

To confirm these results, we examined the effect of deletion of AAN1 on phosphorylation of ribosomal protein S6 (Rps6p), a direct downstream target of TORC1. Since there are two TOR genes in yeast, deletion of TOR1 reduces Rps6p phosphorylation, while treatment with rapamycin fully inhibits TORC1-mediated Rps6p phosphorylation ${ }^{49,50}$. We confirmed that deletion of TOR1 inhibits but does not block Rps6p phosphorylation. Equally important, we find that phosphorylation of Rps6 is indistinguishable in aan $1 \Delta$ and wild-type cells (Fig. 3i,j). Consistent with this, our RNA-Seq studies indicate the gene expression pattern in aan $1 \Delta$ cells is distinct from that observed upon TORC1 inhibition ${ }^{51-57}$. Collectively, these results indicate that Aan1p function is not dependent upon TORC1.

\section{YKL075C targets BCAA metabolism to control actin cytoskeleton structure and function}

Next, we tested whether modulating BCAA levels can drive a change in cytoskeletal stability in wild-type or aan1 1 cells. Here, modulation of BCAAs was conducted in prototrophic yeast strains, which can synthesize BCAAs. Moreover, amino acid depletion or supplementation was carried out for short time periods (20-30 min). Thus, the experimental conditions were designed to alter BCAA levels without eliciting starvation or stress responses.

We found that deletion of BAT2 has no obvious effect on actin cables (Extended Data Fig. $4 a, b)$. On the other hand, bat1 $\Delta$ mutants exhibit an increase in actin cable abundance similar to that observed in aan1 $1 \Delta$ cells (Fig. 4a,b). In addition, we found that short-term (20 min) depletion of leucine from the growth medium does not produce cytoskeletal phenotypes (e.g. global destabilization of the actin cytoskeleton) associated with acute stress. Instead, it results in an increase in actin cable abundance in wild-type cells, reaching the elevated levels observed in aan $1 \Delta$ cells (Fig. 4c,d). Moreover, we found that short-term depletion of another amino acid, lysine, has no detectable effect on actin cable abundance (Extended Data Fig. 4c,d). 
Conversely, leucine supplementation reduced the amount of F-actin in actin cables, as assessed by F-actin staining intensity, in both wild-type and aan $1 \Delta$ cells, and resulted in reduced actin cable length in aan1 $1 \Delta$ cells (Extended Data Fig. 4e-g). It also resulted depolarization of the actin cytoskeleton as assessed by the distribution of actin cables and patches (Extended Data Fig. 4g,h). Finally, we found that deletion of BAT1 or depletion of

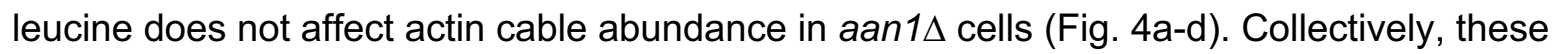
findings support a role for BCAA in modulation of actin cables. They also indicate that Aan $1 p$ and BCAA function in the same pathway for control of actin cable abundance.

Next, we used the Lat-A sensitivity assay described above to determine whether deletion of BAT1 and the associated decrease in BCAA levels affect actin cable stability. We found that actin cable stability is greater in bat $1 \Delta$ cells compared to wild-type cells and similar to that observed in aan $1 \Delta$ and aan $1 \Delta$ bat1 $\Delta$ cells (Fig. 4e,f). Overall, these findings indicate that 1 ) increased actin cable abundance observed in bat $1 \Delta$, aan $1 \Delta$ and aan $1 \Delta$ bat $1 \Delta$ cells may be due to effects on actin cable stability, and 2) BAT1 and AAN1 function in the same pathway for control of actin cable stability.

Finally, we found that deletion of $B A T 1$ and $A A N 1$ also produces similar effects on mitochondrial function. Assessing mitochondrial redox state using mito-roGFP1, we found that mitochondria in bat1 $\Delta$ and aan $1 \Delta$ cells are more reduced and therefore higher functioning compared to mitochondria in wild-type cells. Moreover, we found that the mitochondrial redox state is similar in bat1 $\Delta$ and aan1 $1 \Delta$ cells (Fig. 4 g,h). Finally, higher-functioning mitochondria are preferentially inherited by daughter cells in bat $1 \Delta$ mutants, as in aan $1 \Delta$ and wild-type cells (Extended Data Fig. 4i). These findings indicate that BAT1 and AAN1 have similar effects on mitochondrial function: deletion of either gene promotes mitochondrial function but has no effect on asymmetric inheritance of the organelle. 


\section{Discussion}

Age-associated declines in actin cytoskeletal function occur in mammalian cells, tissues and organs. However, the mechanism underlying this process is not well understood. Here, we report that the stability of actin cables, essential components of the yeast actin cytoskeleton, declines with age. We identified a previously undescribed open reading frame, which we refer to as $A A N 1$, that impacts on actin cable stability, mitochondrial function, BCAA metabolism and cellular lifespan. Moreover, we obtained evidence that modulation of BCAA metabolism or levels of leucine affects actin cable stability and abundance. Finally, we found that AAN1 function is not dependent upon the TORC1 pathway for sensing nutrients and regulating lifespan.

The stability of the actin cytoskeleton declines with age in yeast: Our studies focused on actin cables, essential components of the yeast actin cytoskeleton that serve as tracks and movement generators for organelles and other cargoes during asymmetric yeast cell division. Actin cables assemble at sites of polarized cell surface growth in the bud by direct interactions with the polarisome, a protein complex that stimulates actin polymerization for actin cable assembly ${ }^{58,59}$. Polarisome-dependent insertion of newly polymerized actin into the end of actin cables drives elongation of actin cables and promotes extension of those structures from the bud into the mother cell.

We detected age-linked declines in the amount of actin polymer within actin cables. Consistent with this, we observed an increase in the sensitivity of actin cables to destabilization by Lat-A in old yeast (>10 generations in replicative aging) compared to young yeast. These findings indicate that actin cable stability declines with age in yeast. We also detect defects in actin cable organization in yeast as they undergo replicative aging. Specifically, we detect depolarized actin cables, which do not align along the mother-bud axis, in cells of advanced age. This defect in actin cable polarization may reflect age-linked alterations in cell polarity. 
Alternatively, they may reflect fragmentation of destabilized actin cables, and release of those fragments from their interactions with the polarisome and cell polarity machinery of yeast. These models are not mutually exclusive.

Recent studies in Caenorhabditis elegans revealed age-associated declines in the integrity of the actin cytoskeleton in muscle, intestine and hypodermis, and a role for PAT-10, a calcium-binding actin-associated protein, in that process ${ }^{60,61}$. Interestingly, deleting pat-10 destabilizes the actin cytoskeleton and results in premature aging; while pat-10 overexpression has the opposite effect ${ }^{61}$. Since essential elements of the yeast actin cytoskeleton also undergo a decrease in stability with age, our findings indicate that actin cytoskeletal destabilization may be a conserved aging phenotype in eukaryotic cells.

\section{Identification of a novel regulator of actin cables, mitochondria and lifespan in yeast: We} carried out a screen of a library of 4,848 yeast strains, which each bear a deletion of a nonessential yeast gene, for strains that exhibit reduced sensitivity to Lat-A. The concentration of Lat-A used for the screen destabilizes actin cables and inhibits yeast cell growth, but does not affect other actin-containing structures. Actin cable components and assembly factors were not identified as hits in this screen. Deletion of these proteins destabilizes actin cables and is expected to increase Lat-A sensitivity. Instead, the screen revealed 18 gene deletions that promote yeast growth in the presence of low levels of Lat-A. 13 of these deletion strains also exhibit an increase in actin cable abundance in the absence of Lat-A. GO term analysis revealed that hits that impact on Lat-A sensitivity and actin cable abundance function in amino acid metabolism and endocytosis. This raises the possibility that other hits identified in our screen may contribute to Aan $1 \mathrm{p}$ function in modulation of the actin cytoskeleton in response to BCAA.

Our efforts focused on the previously uncharacterized open reading frame YKL075C, which we refer to as $A A N 1$. Deletion of $A A N 1$ results in an increase in actin cable abundance. It 
also results in reduced sensitivity of actin cables to destabilization by treatment with Lat-A.

Finally, we found that deletion of $A A N 1$ results in extended replicative lifespan and increased healthspan as assessed by improved mitochondrial redox state and reduced mean generation time during cell growth. Thus, we obtained evidence for a role for AAN1 in actin cable stability and abundance, mitochondrial quality control and replicative lifespan in yeast.

Our previous studies revealed a role for actin cables in control of lifespan through effects on mitochondrial quality control. Specifically, we found that promoting actin cable dynamics results in inheritance of higher-functioning mitochondria by yeast daughter cells, which promotes cell fitness and lifespan ${ }^{20}$. Although deletion of $A A N 1$ promotes mitochondrial function, extends replicative lifespan and affects actin cable stability, there was no effect on the rate of retrograde actin cable flow. Thus, the increase in mitochondrial function and lifespan observed in aan $1 \Delta$ cells is not due to effects on actin cable dynamics.

Moreover, although deletion of $A A N 1$ promotes mitochondrial function, it is possible that other actin cable-dependent aging determinants also contribute to AAN1 effects on lifespan. For example, defects in the polarisome or destabilization of the actin cytoskeleton results in premature aging in yeast, by inhibiting retention of protein aggregates, an aging determinant, in the mother cell ${ }^{17,18}$. Actin cables also serve as tracks for inheritance of vacuoles in yeast ${ }^{62}$ and inheritance of more acidic, higher-functioning vacuoles has also been linked to mitochondrial function and lifespan control in yeast ${ }^{22}$. Thus, AAN1 effects on actin cables may impact on lifespan through effects on mitochondria and other aging determinants.

Finally, previous studies also support a role for the actin bundling protein, Scp1p, in mitochondrial quality control, actin dynamics, and lifespan control ${ }^{63}$. Since Scp $1 p$ localizes to actin patches, it affects actin dynamics on structures other than actin cables. Moreover, Scp1p affects lifespan in stationary phase, not dividing yeast cells, whereas Aan1p affects lifespan in dividing yeast cells. Thus, available evidence indicates that Aan1p and Scp1p affect lifespan by 
different cytoskeleton-based mechanisms. These observations underscore the complexity of the actin cytoskeleton and its impact on mitochondria and lifespan.

\section{BCAAs are regulated by Aan1p and control actin cable abundance and stability: Aan $1 p$}

has no conserved protein domains or no homology to any known protein. It localizes to punctate cytoplasmic structures that do not colocalize with actin cables or actin patches. Analysis of the transcription signature of $A A N 1$ deletion cells revealed an unexpected link between $A A N 1$ and BCAA metabolism: deleting AAN1 decreases the expression of BCAA biosynthetic genes, and increases the expression of a BCAA degradation gene. Moreover, we find decreasing BCAA biosynthesis by deleting BAT1 or depleting a specific BCAA, leucine, in wild-type cells produces an increase in actin cable abundance and stability that resembles that observed in aan $1 \Delta$ cells. Finally, reducing BCAA, by deletion of a BCAA biosynthetic enzyme or leucine depletion, did not affect actin cable abundance in aan $1 \Delta$ cells.

An excess of BCAAs is toxic and is the basis for Maple Syrup Urine Disease, an inherited metabolic disorder that is characterized by neuronal dysfunction and cerebral atrophy ${ }^{64}$. Previous studies indicate that leucine supplementation results in actin-dependent changes in the morphology of astrocytes ${ }^{64}$. Similarly, in yeast, we find that short-term supplementation with leucine results in destabilization of actin cables and changes in actin cable morphology, in wild-type and aan $1 \Delta$ cells. We also provide the first evidence that depletion of leucine can promote actin cable abundance and stability and that Aan $1 p$ function in control of actin cables is dependent on its function in regulating BCAAs. However, leucine supplementation is required for chronological lifespan extension in non-dividing yeast cells ${ }^{65,66}$. Thus, leucine effects on processes including lifespan control are complex and may be influenced by dose and context.

The mechanism of action of Aan1p remains to be determined. Aan1p does not localize to actin cables, mitochondria or the nucleus. Therefore, it has indirect effects on the actin 
cables, mitochondrial function and BCAA gene expression. Moreover, we find that Aan1p function is not dependent upon TORC1: deletion of AAN1 does not affect the phosphorylation of a conserved TORC1 substrate or recovery from rapamycin. It also does not produce a pattern of gene expression observed upon TORC1 activation. However, Aan1p may affect the General Amino Acid Control pathway (GAAC), a signaling pathway that regulates yeast cell growth, metabolism, translation and lifespan in response to amino acid limitations. Indeed, GAAC affects expression of amino acid biosynthesis genes, including BAT1, and aminoacyl-tRNA synthetase ${ }^{67}$. Moreover, the actin cytoskeleton has been linked to GAAC function in translational regulation $^{68-71}$. Ongoing studies focus on the mechanisms underlying AAN1 control of BCAA metabolism, and how BCAAs control the actin cytoskeleton and lifespan in yeast. Finally, the links between Aan1p, nutrient sensing, mitochondria and the actin cytoskeleton may impact on health and disease, including cancer cell metabolism and migration. 
bioRxiv preprint doi: https://doi.org/10.1101/2021.10.07.463535; this version posted October 7, 2021. The copyright holder for this preprint (which was not certified by peer review) is the author/funder, who has granted bioRxiv a license to display the preprint in perpetuity. It is made available under aCC-BY-NC-ND 4.0 International license.

\section{Figures}
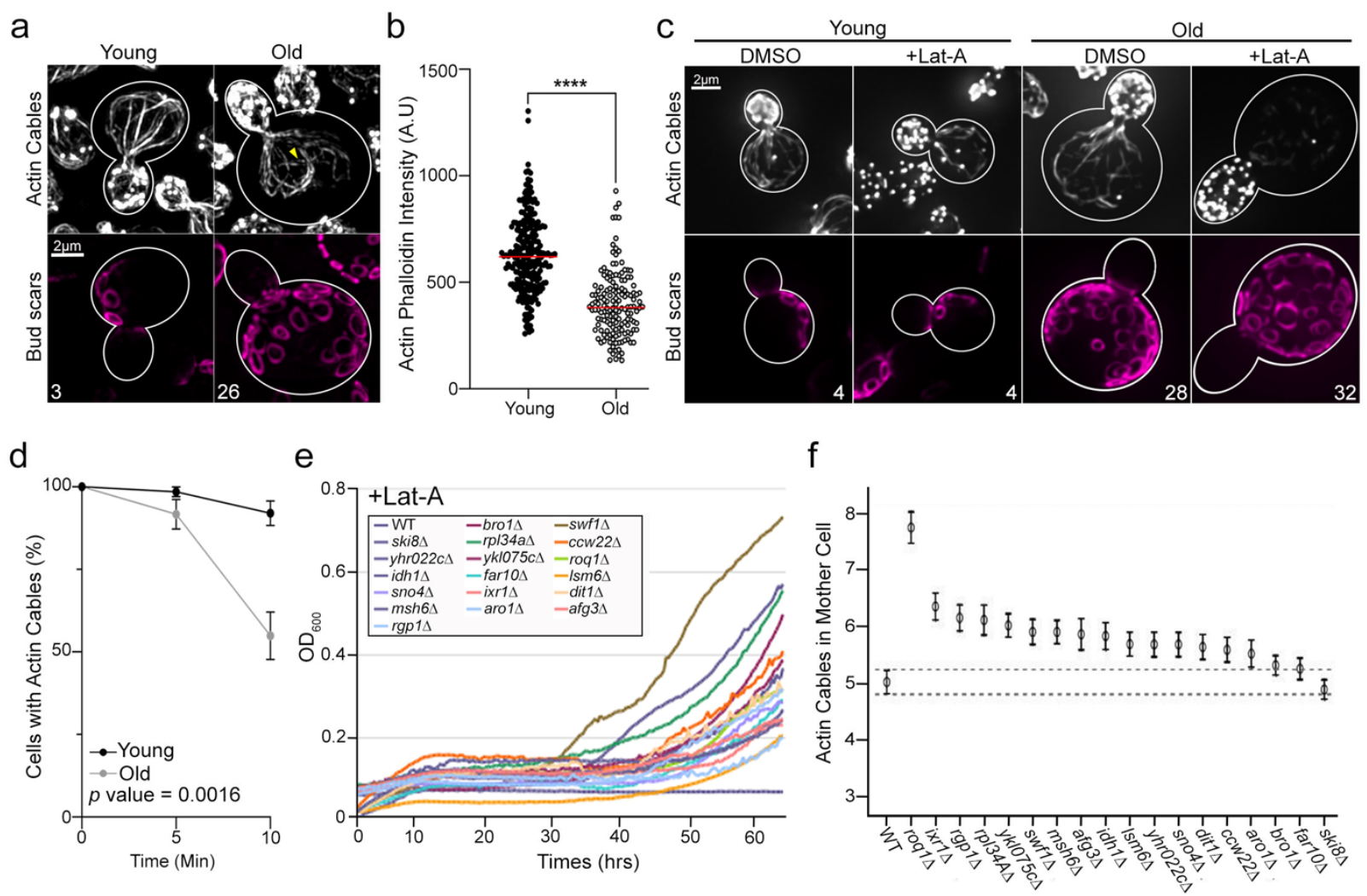


\section{Fig. 1: Actin stability declines with age.}

a, Representative SIM images of F-actin and bud scars of young and old wild-type (WT) cells stained with Alexa488-phalloidin and Alexa594-wheat germ agglutinin (WGA594), respectively. Yellow arrow identifies a depolarized actin cable. b, Quantification of Alexa488-phalloidin corrected mean signal intensity in mother cells of young and old cells isolated from mCAD. Combined $n$ of cells/strain from 3 trials: 248 (young) and 152 (old). The $p$ value $(<0.0001)$ was determined by a two-tailed non-parametric Mann-Whitney test. c, Representative SIM images of Alexa488-phalloidin-stained actin and Alexa594-WGA-stained bud scars in young and old WT cells treated with vehicle (DMSO) or $1 \mu \mathrm{M}$ Lat-A for 10 mins. d, Time-course of actin cable loss during treatment with $1 \mu \mathrm{M}$ Lat-A. Error bars: SEM of 3 trials. $(n \geq 126$ cells/timepoint/condition/trial). e, Growth curves of deletion strains that exhibit reduced sensitivity to Lat-A $(10 \mu \mathrm{M})$ in our genome-wide screen. $\mathbf{f}$, Actin cable abundance in WT cells and the 13 hits from the screen. Actin was visualized by rhodamine-phalloidin staining and widefield fluorescence microscopy. Scale bars, $2 \mu \mathrm{m}$. 
a

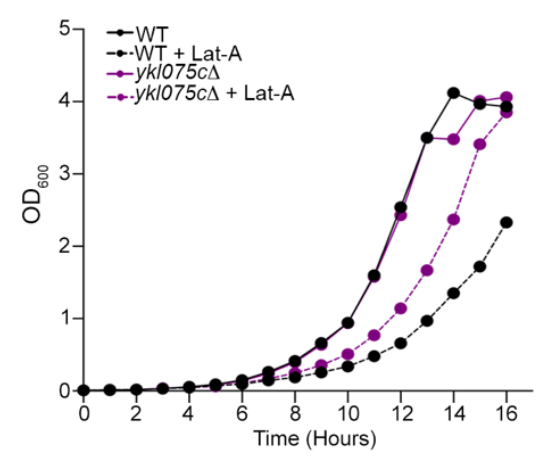

b

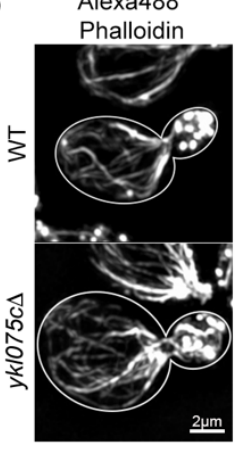

C

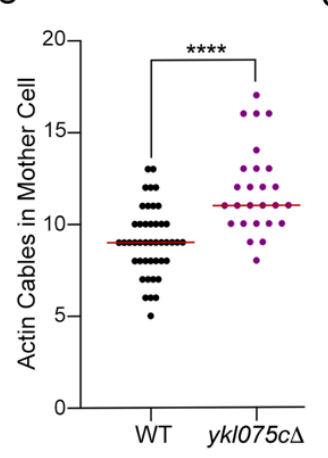

d

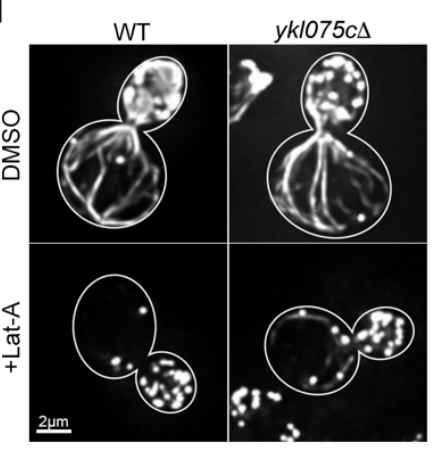

g

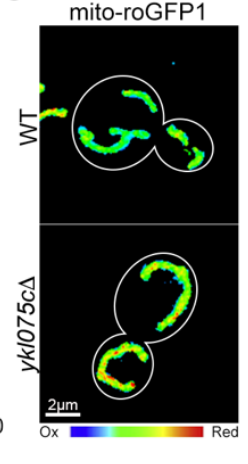

h

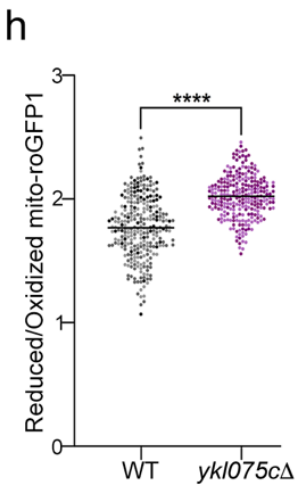

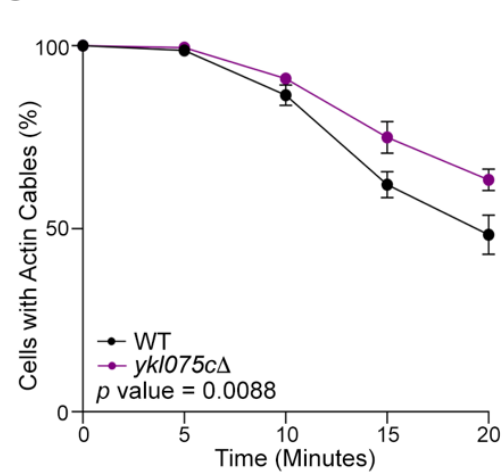

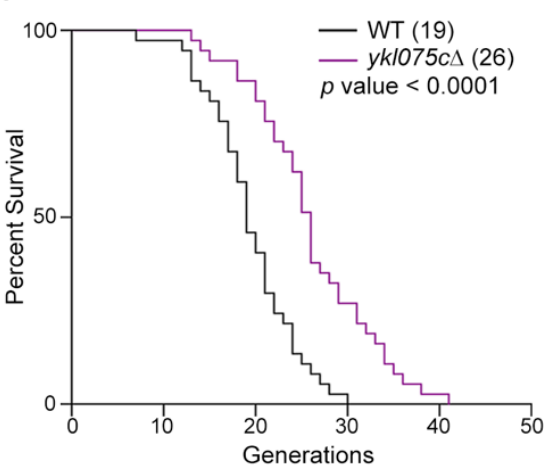


Fig. 2: Identification of a gene (YKL075C) that affects actin cable stability, mitochondria, and lifespan.

a, Growth curves of WT and $y k 1075 c \Delta$ cells treated with vehicle (DMSO) or $2 \mu \mathrm{M}$ Lat-A for $16 \mathrm{~h}$.

Data from a representative trial is shown ( $n=3$ trials). b, Representative images of Alexa488phalloidin stained F-actin of WT and $y k 1075 c \Delta$ cells. c, Actin cable abundance in mother cells of mid-log phase WT and $y k 1075 c \Delta$ cells. $n=44$ cells $(\mathrm{WT})$ and 26 cells $(y k 1075 c \Delta)$. Data from a representative trial is shown $(n=3$ trials $) . p$ value $(<0.0001)$ was determined by a two-tailed non-parametric Mann-Whitney test. d, Representative images of Alexa488-phalloidin stained Factin of WT and $y k / 075 c \Delta$ cells treated with vehicle (DMSO) (top panels) or $2 \mu \mathrm{M}$ Lat-A for 20 min (bottom panels). e, Quantitation of actin cables in WT and $y k 1075 c \Delta$ cells during treatment with $2 \mu \mathrm{M}$ Lat-A. Error bars: SEM of 5 trials ( $n \geq 256$ cells/strain/timepoint). $p$ value (0.0088) was determined by simple linear regression analysis. f, RLS of WT and $y k 1075 c \Delta$ cells determined by manual micromanipulation. $n$ of cells/strain: 35 (WT) and $36(y k / 075 c \Delta) . p$ value $(<0.0001)$ was determined by the Mantel-Cox test. Data from a representative trial is shown ( $n=3$ trials). $\mathbf{g}$, Representative images of mitochondrial redox state (reduced:oxidized ratio) visualized using mdito-roGFP1 in WT and $y$ kl075c $\Delta$ cells. Higher numbers/warmer colors indicate more reducing mitochondria. h, Quantification of mito-roGFP1 redox ratios in WT and $y k 1075 c \Delta$ cells.

Combined $n$ of cells/strain from 3 trials: 284 (WT) and $260(y k l 075 c \Delta)$. $p$ value $(<0.0001)$ was determined by a two-tailed non-parametric Mann-Whitney test. Scale bars, $2 \mu \mathrm{m}$. 


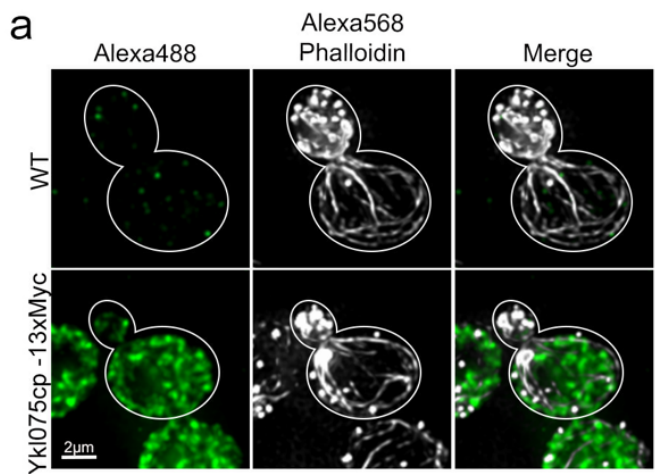

b

C

\begin{tabular}{|c|c|c|}
\hline \multicolumn{3}{|c|}{$y k / 075 c \Delta$ Cells } \\
\hline \multicolumn{3}{|c|}{ BCAA Metabolism } \\
\hline $\begin{array}{c}\text { Systematic } \\
\text { Name }\end{array}$ & $\begin{array}{c}\text { Gene } \\
\text { Name }\end{array}$ & $\begin{array}{c}\text { FC } \\
\text { (log } 2)\end{array}$ \\
\hline YGL009C & BAT1 & \\
\hline YHR208W & BAT2 & \\
\hline YDR380W & ILV2 & \\
\hline YBR145W & ILV3 & \\
\hline YMR108W & ILV5 & \\
\hline YJR016C & ILV6 & \\
\hline YLR355C & LEU1 & \\
\hline YJR148W & LEU4 & \\
\hline YOR108W & LEU9 & \\
\hline
\end{tabular}

g

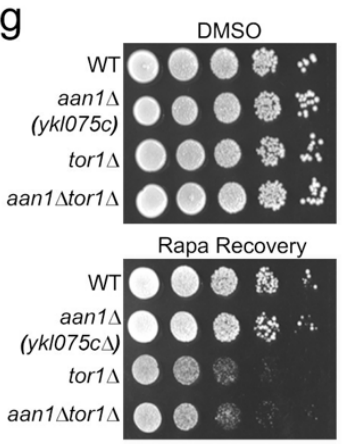

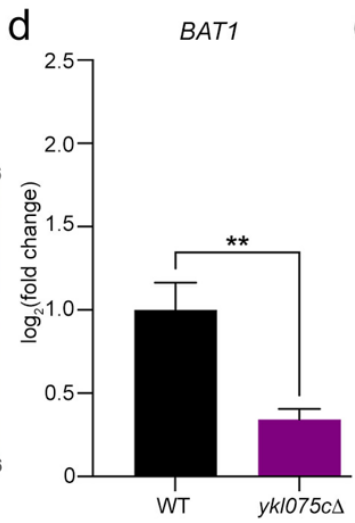

$\mathrm{h}$

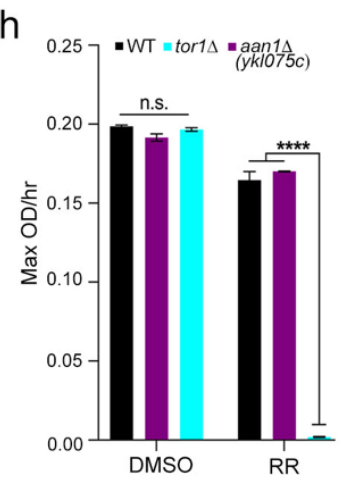

BCAA biosynthetic processCellular amino acid biosynthetic procees Siderophore transmembrane transport-

Valine biosynthetic process Arginine biosynthetic processIsoleucine biosynthetic process Mitochondrial electron transportCarboxylic acid metabolic processDrug exportIron ion homeostasis Ion transportLeucine biosynthetic processSiderophore transportCellular iron ion homeostasis Response to drug
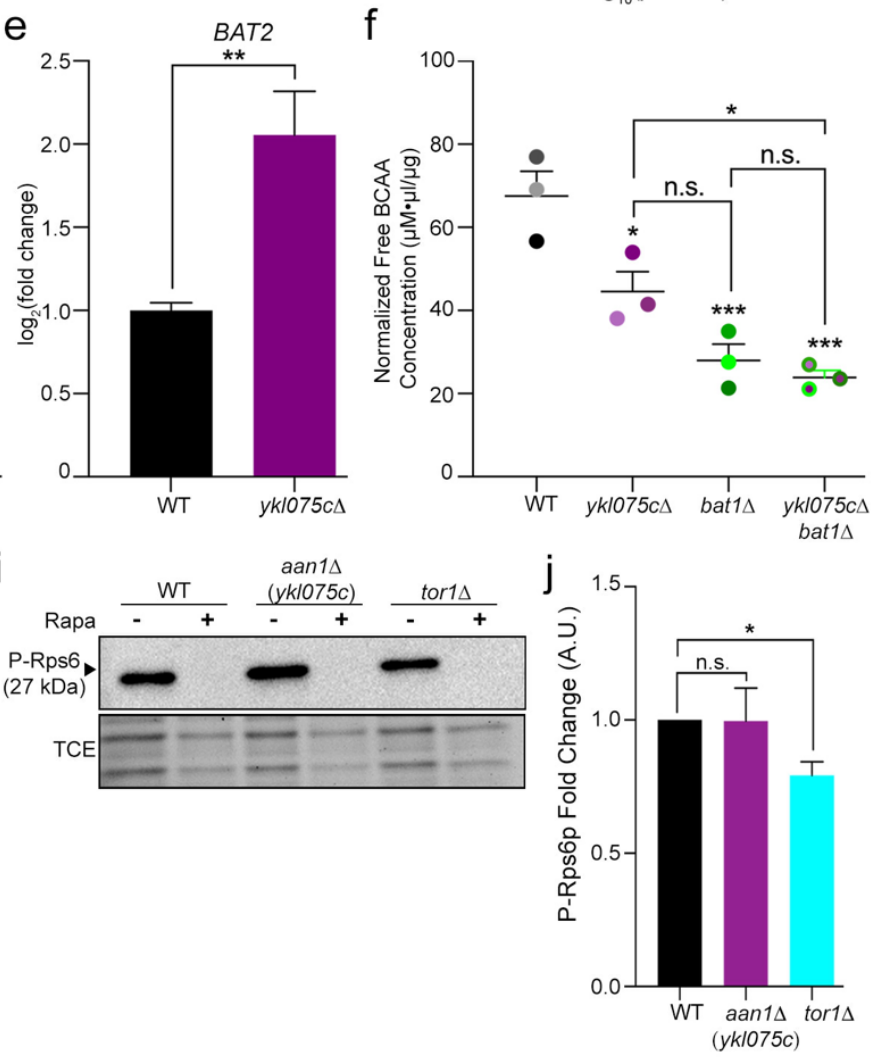

\section{Fig. 3: YkI075cp protein localization and links to branched-chain amino acid (BCAA)}

\section{metabolism.}

a, Localization of YkI075cp and the actin cytoskeleton in cells containing either native YKL075C or YKL075C tagged with the Myc epitope. Ykl075cp-13Myc visualized by immunofluorescence using anti-Myc antibodies and Alexa488-tagged secondary antibodies. The actin cytoskeleton was visualized by staining with Alexa568-phalloidin. Scale bar, $2 \mu \mathrm{m}$. b, Top 15 gene ontology (GO) terms with significant gene expression changes in $y k 1075 c \Delta$ cells. GO terms are arranged 
on the $y$ axis and significance is plotted on the $x$ axis. c, Table of BCAA proteins with altered transcript levels in $y k 1075 c \Delta$ cells. d,e, Validation of mRNA transcript levels of BCAA transaminases $B A T 1$ and $B A T 2$ by quantitative PCR. Decreased BAT1 and increased BAT2 gene expression in $y k 1075 c \Delta$ cells are significantly different from WT cells (unpaired two-tailed ttest; $p$ values: 0.0029 (WT vs. bat1 $\Delta$ ) and 0.0033 (WT vs. bat2 $\Delta$ )). Data is representative of 3 trials. f, Quantification of free intracellular BCAA levels in WT, yk1075cs, bat1 $\Delta$, and $y k 1075 c \Delta$ bat1 1 cells using Cell BioLabs BCAA colorimetric ELISA kit. Intracellular free BCAA levels were calculated based on a leucine standard and normalized to protein concentration isolated from each strain. Data values are represented from 3 trials and statistically analyzed using a twotailed one-way ANOVA test $\left({ }^{*} p<0.05,{ }^{* *} p<0.01,{ }^{* * *} p<0.001 ;\right.$ n.s., not significant $) . p$ values:

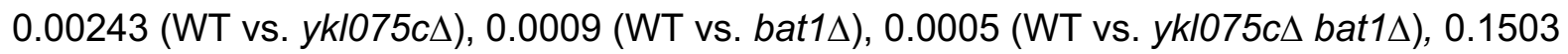

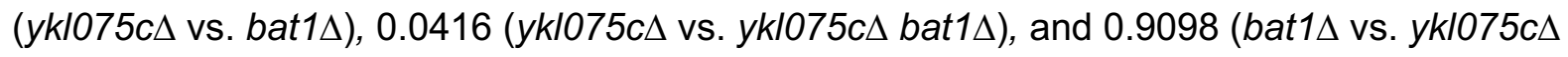
bat1 $1 \Delta) . \mathbf{g}$, Serial dilutions showing growth on YPD plates of WT, aan1 $(y k / 075 c) \Delta$, tor $1 \Delta$, and aan1 $\Delta$ tor1 $\Delta$ cells recovering from either DMSO or $200 \mathrm{nM}$ rapamycin treatment. $\mathbf{h}$, Growth rate $\left(\mathrm{OD}_{600} / \mathrm{h}\right.$ for $\left.72 \mathrm{~h}\right)$ of $\mathrm{WT}$, aan1(ykl075c) $\Delta$, tor1 $\Delta$, and aan1 $\Delta$ tor1 $\Delta$ cells during recovery from DMSO or $200 \mathrm{nM}$ rapamycin treatment (RR). i, Representative western blot of the phosphorylation of ribosomal protein-S6 (Rps6p) in mid-log phase WT, aan1(ykl075c) $\Delta$, and tor1 $\Delta$ cells in the presence or absence of rapamycin $(200 \mu \mathrm{M})$. TCE, total protein loading control. j, Quantification of Rps6p phosphorylation from western blot analysis of untreated or rapamycin-treated WT, aan1 $(y k 1075 c) \Delta$, and tor $1 \Delta$ cells. Data are representative of 3 trials. $p$ value: 0.035 (non-parametric Kruskal-Wallis test). 
bioRxiv preprint doi: https://doi.org/10.1101/2021.10.07.463535; this version posted October 7, 2021. The copyright holder for this preprint (which was not certified by peer review) is the author/funder, who has granted bioRxiv a license to display the preprint in perpetuity. It is made available under aCC-BY-NC-ND 4.0 International license.
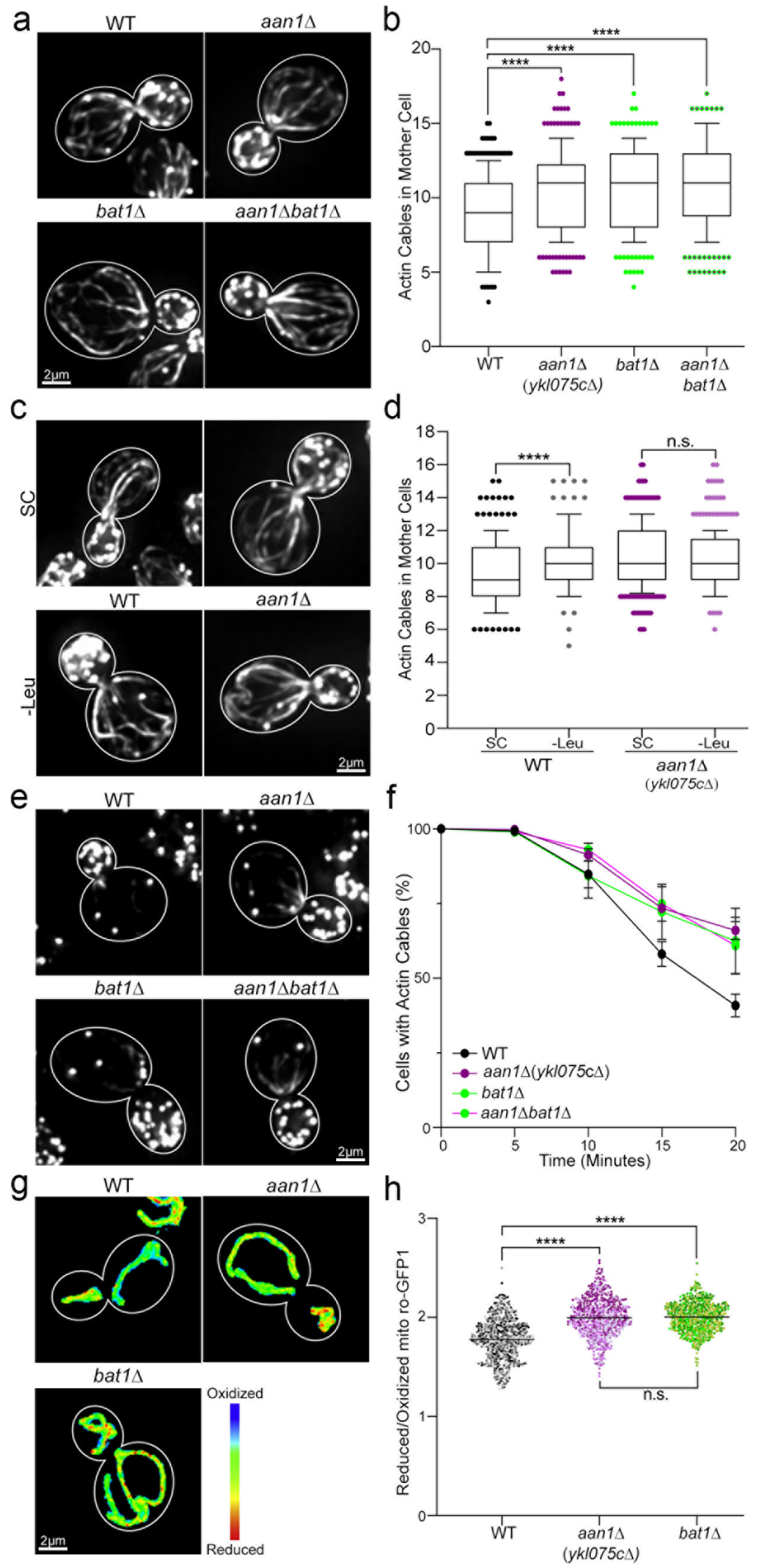
Fig. 4: AAN1(YKL075C) affects actin cables and mitochondria through its function in BCAA homeostasis.

a, Representative images of Alexa488-phalloidin stained F-actin of mid-log phase WT, aan1 $\Delta$, bat $1 \Delta$, and aan $1 \Delta$ vbat1 $\Delta$ cells. b, Actin cable abundance in the strains shown in panel (a).

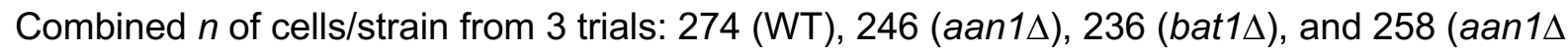

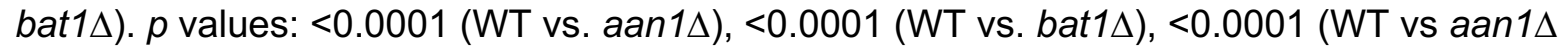

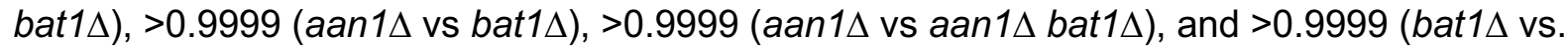
aan1 $\Delta$ bat1 $\Delta$ ). $p$ values were generated from two-tailed non-parametric Kruskal-Wallis tests. c, Representative images of AlexaFluor488-phalloidin stained F-actin of mid-log phase WT, aan $1 \Delta$, bat1 $\Delta$ or aan $1 \Delta$ bat1 $1 \Delta$ cells grown in synthetic complete (SC) medium or SC without leucine (-Leu). d, Actin cable abundance in the presence or absence of leucine. Combined $n$ of

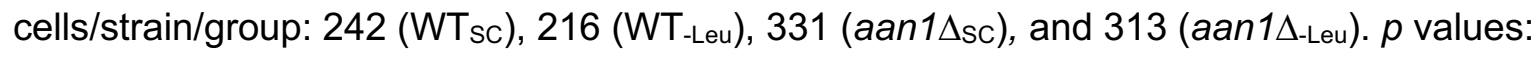

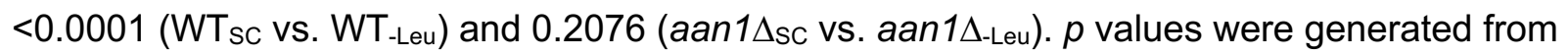
two-tailed non-parametric Kruskal-Wallis test of 3 trials. e, Representative images of Alexa488phalloidin stained F-actin of mid-log phase WT, aan $1 \Delta$, bat $1 \Delta$, and aan $1 \Delta$ bat $1 \Delta$ cells treated with $2 \mu \mathrm{M}$ Lat-A for 20 min. f, Actin cable loss as a function of the time of Lat-A treatment in WT, aan1 $\Delta$, bat1 $\Delta$, and aan1 $\Delta$ bat1 $\Delta$ cells. Error bars: SEM of 3 trials $(n=100$ cells/strain/trial). $p$

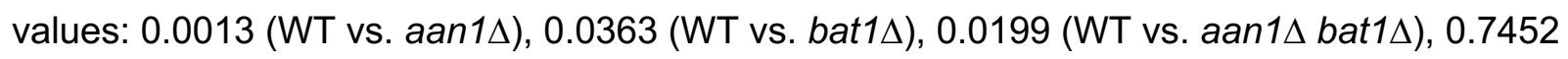
(aan1 $\Delta$ vs. bat1 $\Delta$ ), 0.6833 (aan1 $\Delta$ vs. aan $1 \Delta$ bat1 $\Delta$ ), and 0.9868 (bat1 $\Delta$ vs. aan1 $\Delta$ bat1 $\Delta) . p$ values were determined by simple linear regression analysis. $\mathbf{g}$, Representative images of mitochondrial redox state visualized using mito-roGFP1. h, Reduced:oxidized mito-roGFP1 ratios in WT, aan1 $1 \Delta$, bat1 $\Delta$, and aan $1 \Delta$ bat1 $\Delta$ cells. Combined $n$ of cells/strain of 3 trials: 546

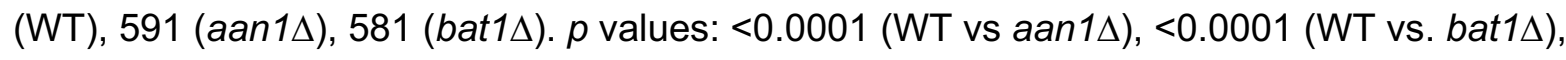
and $>0.9999$ (aan1 $1 \Delta$ vs. bat1 $\Delta$ ). $p$ values were determined using a two-tailed non-parametric Kruskal-Wallis test. Scale bars, $2 \mu \mathrm{m}$. 
a

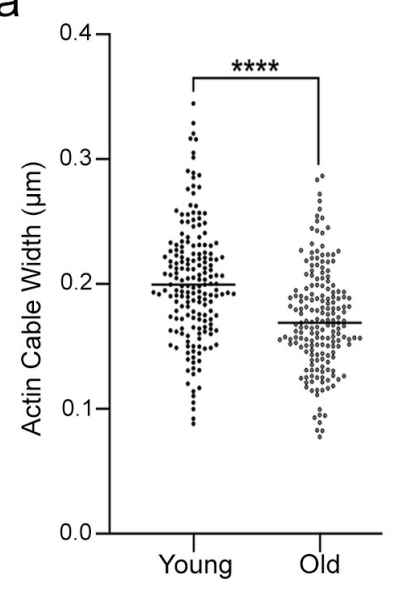

d

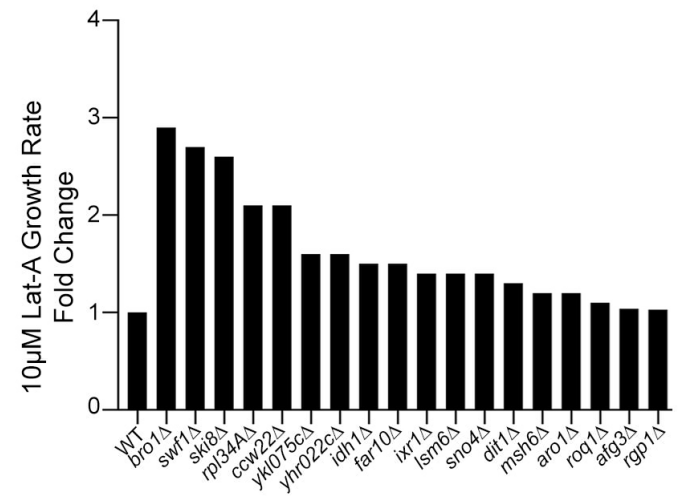

b

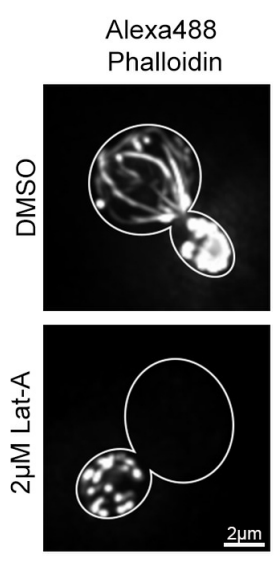

e

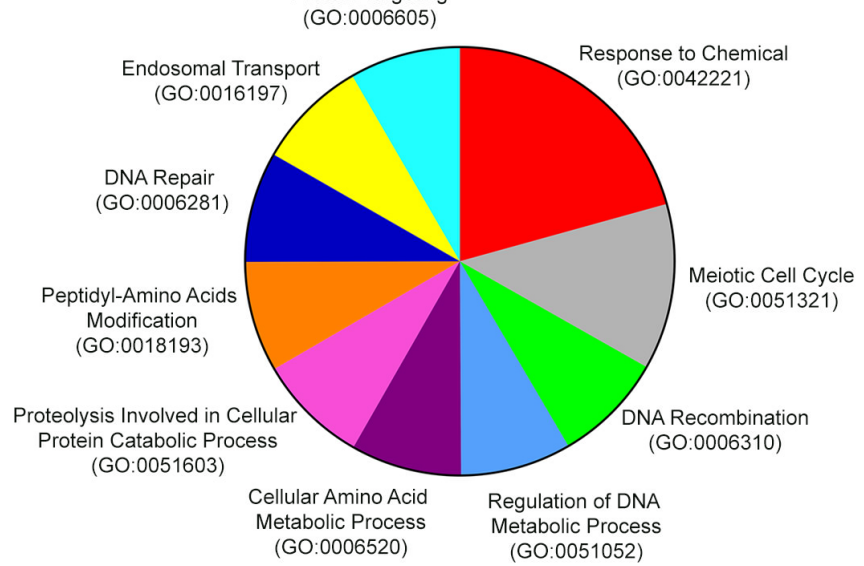

Extended Data Fig. 1: Phenotypic analysis of aging effects on actin cables and of the hits identified in the screen for actin cable modulators.

a, Quantification of apparent actin cable width in young and old WT cells. Data from representative trial. $\mathrm{n}$ of cells/strain: 183 (young) and 184 (old) cells. $p$ value: $<0.0001$ (nonparametric Mann-Whitney test). b, Representative images of Alexa488-phalloidin stained actin structures in mid-log phase WT cells treated with vehicle (DMSO) or $2 \mu \mathrm{M}$ Lat-A for 20 mins. c, Growth curves of hits from the screen in the presence of vehicle (DMSO). d, Growth rate of deletion strains relative to WT cells upon treatment with $10 \mu \mathrm{M}$ Lat-A. e, Gene ontology (GO) terms from the biological process Ontology of current annotated genes identified as hits in the 
bioRxiv preprint doi: https://doi.org/10.1101/2021.10.07.463535; this version posted October 7, 2021. The copyright holder for this preprint

(which was not certified by peer review) is the author/funder, who has granted bioRxiv a license to display the preprint in perpetuity. It is made available under aCC-BY-NC-ND 4.0 International license.

Lat-A screen determined using SGD GO SLIM. GO terms are shown as percent to the total ORFs for GO terms associated with 2 or more hits. 


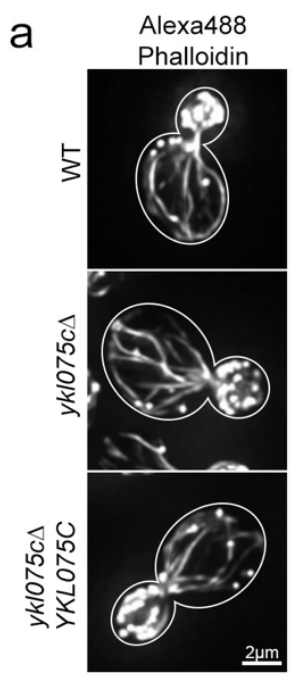

b

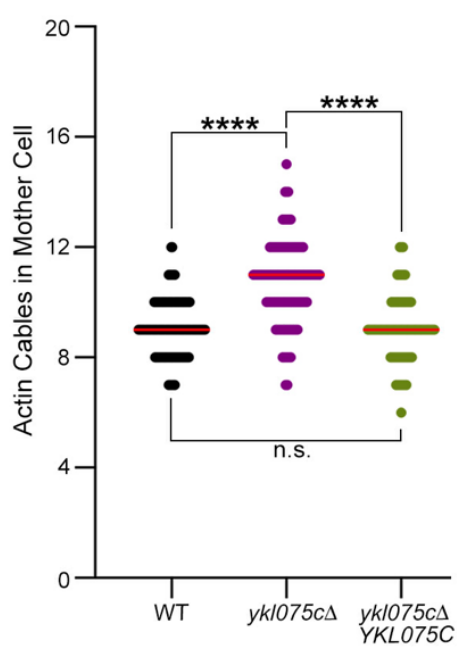

C

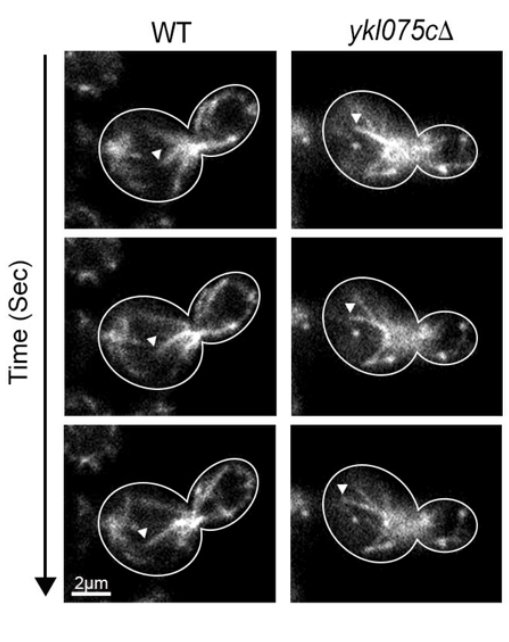

d

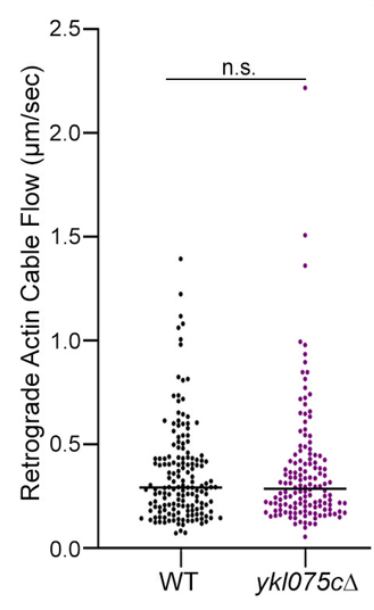

e

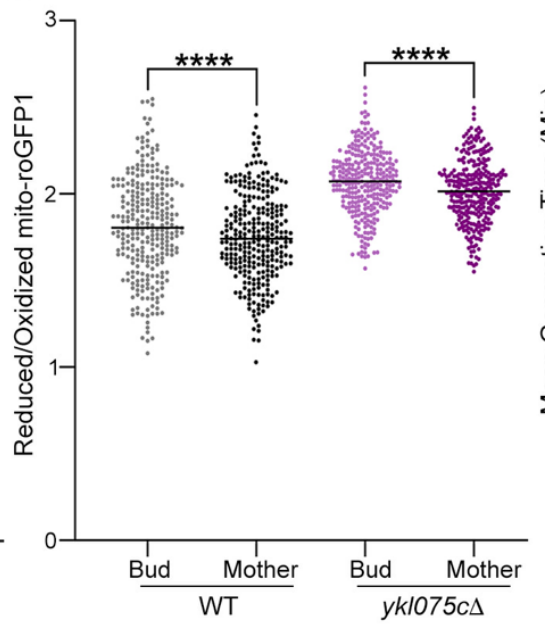

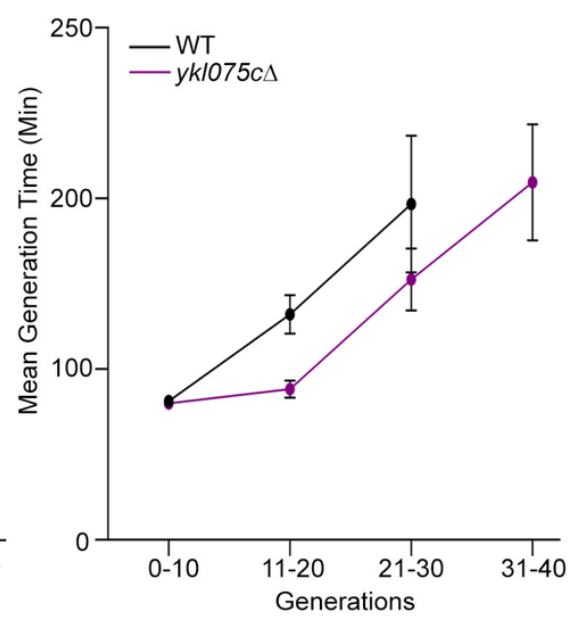

Extended Data Fig. 2: Phenotypic analysis of the actin cytoskeleton and mitochondrial quality in $y k 1075 c \Delta$ cells.

a, Representative images of Alexa488 phalloidin-stained F-actin of mid-log phase WT and $y k 1075 c \Delta$ cells and $y$ k1075c $\Delta$ cells that express YKL075C. b, Quantification of actin cable abundance in WT, $y k 1075 c \Delta$, and $y k 1075 c \Delta Y K L 075 C$ cells. Combined $n$ of cells/strain of 3 trials:

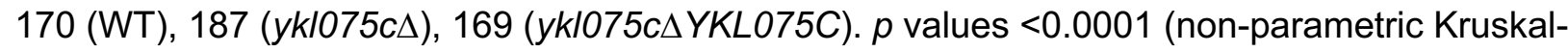
Wallis test). c, Time-lapse frames showing retrograde actin cable flow (RACF) visualized using Abp140p-GFP in mid-log phase WT and $y k 1075 c \Delta$ cells. Arrowheads point to a motile actin 
cable. d, Quantification of RACF rates in WT and $y k 1075 c \Delta$ cells. Combined $n$ of cells/strain of 3

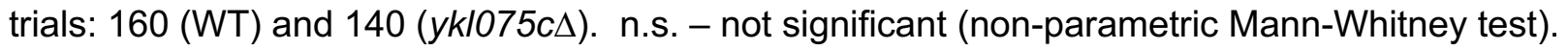
e, Reduced:oxidized mito-roGFP1 ratios of mitochondria in mother cells and buds of mid-log phase WT and $y k 1075 c \Delta$ cells. Combined $n$ of cells/strain of 3 trials: 284 (WT bud and mother), 300 (ykl075cs bud and mother). $p$ value $<0.0001$ (Wilcoxon matched-pair test). f, Quantification of mean generation time (time between emerging consecutive buds from the same mother) of WT and $y k 1075 c \Delta$ cells during RLS analysis. Error bars: SEM, $\mathrm{n}=\geq 35$ cells/strain/trial for 3 trials. 
a

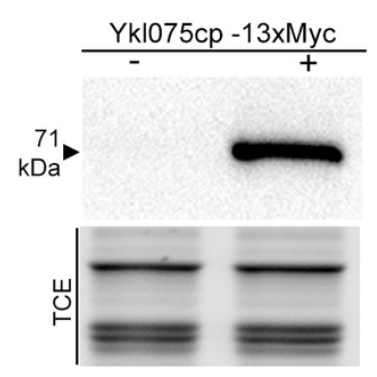

b

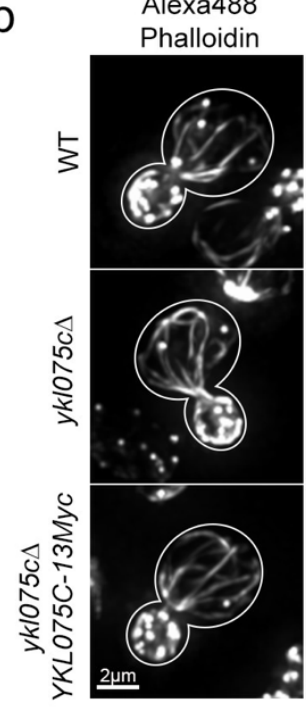

C

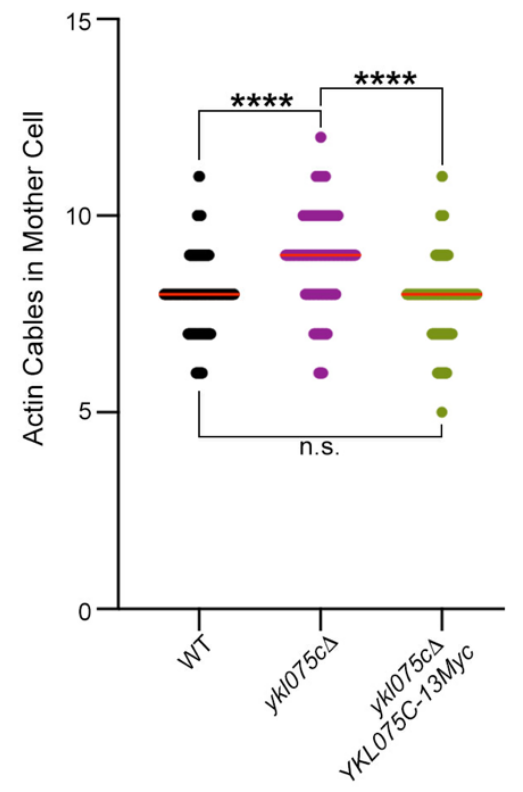

Extended Data Fig. 3: Analysis of Yk1075cp-13Myc for cellular localization.

a, Western blot of Ykl075cp (Aan1p) tagged at its C-terminus with 13 copies of the Myc epitope.

TCE, total protein loading control. b, Representative images of Alexa488 phalloidin-stained Factin of mid-log phase WT and $y k 1075 c \Delta$ cells, and $y k 1075 c \Delta$ that express YKLO75C-13Myc. c, Quantification of actin cable abundance in WT, $y$ kl075c , and $y k 1075 c \Delta$ YKLO75C-13Myc cells.

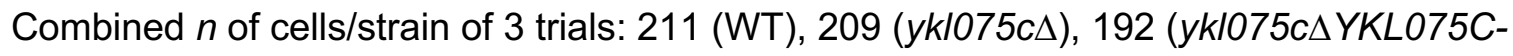
$13 M y c) . p$ values $<0.0001$ (non-parametric Kruskal-Wallis test). 

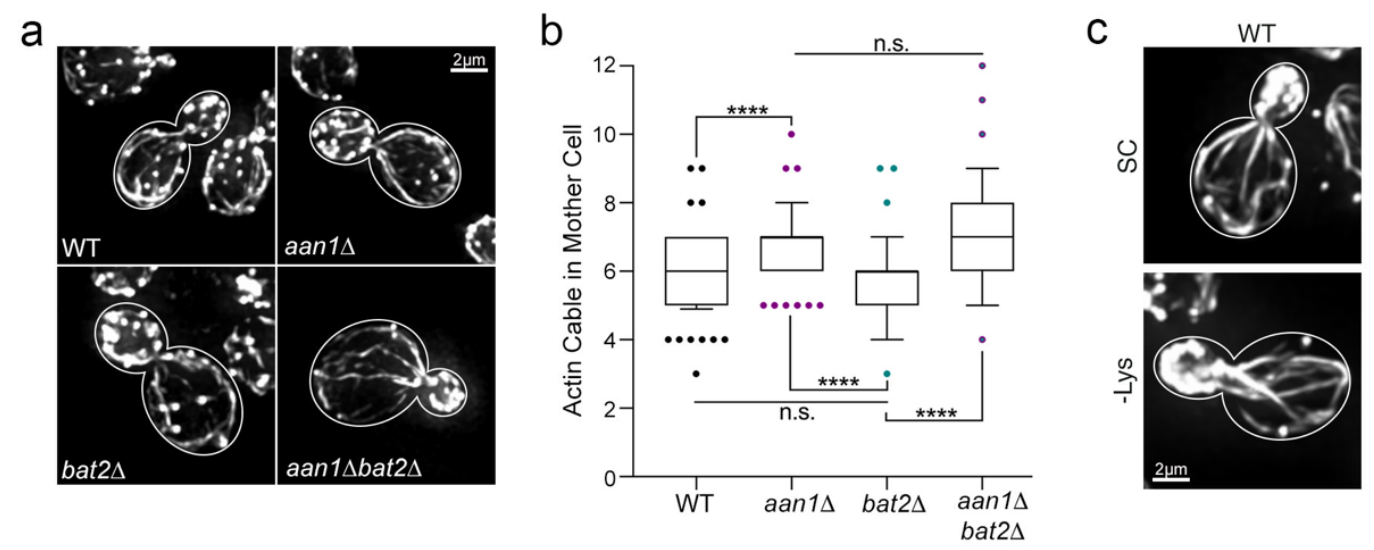

d

e

f
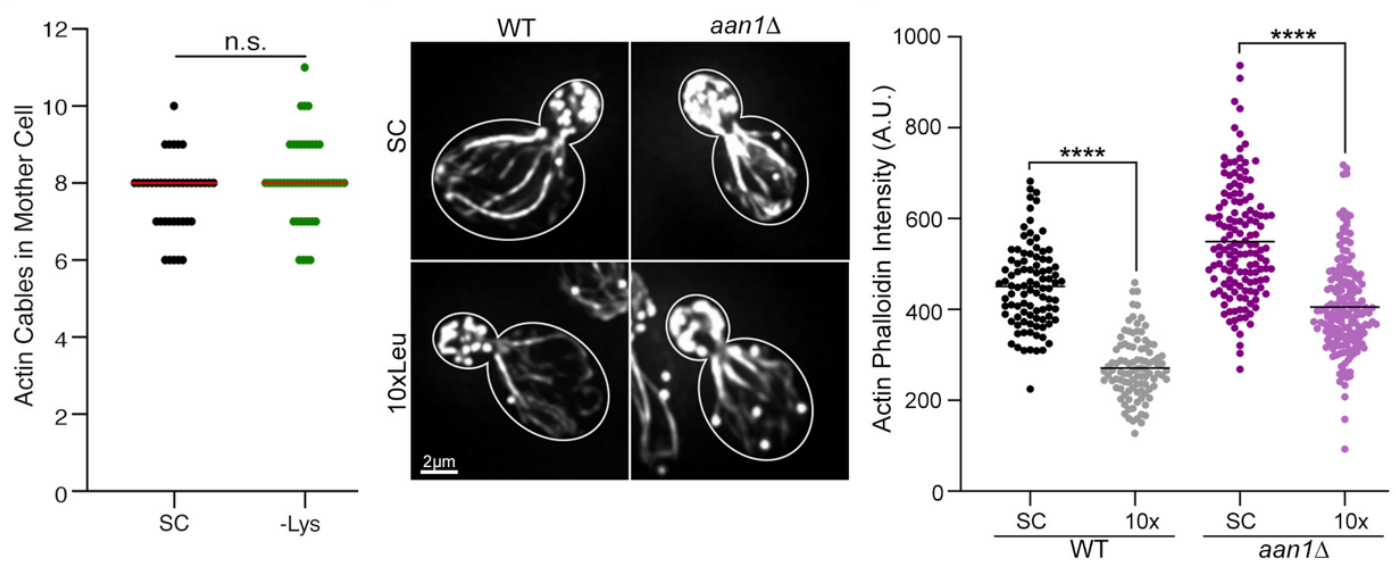

g

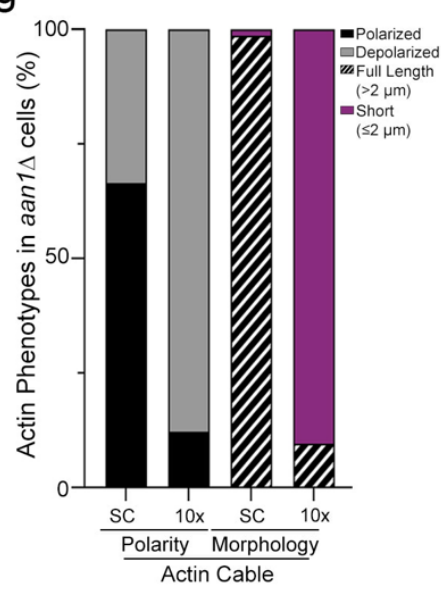

$\mathrm{h}$
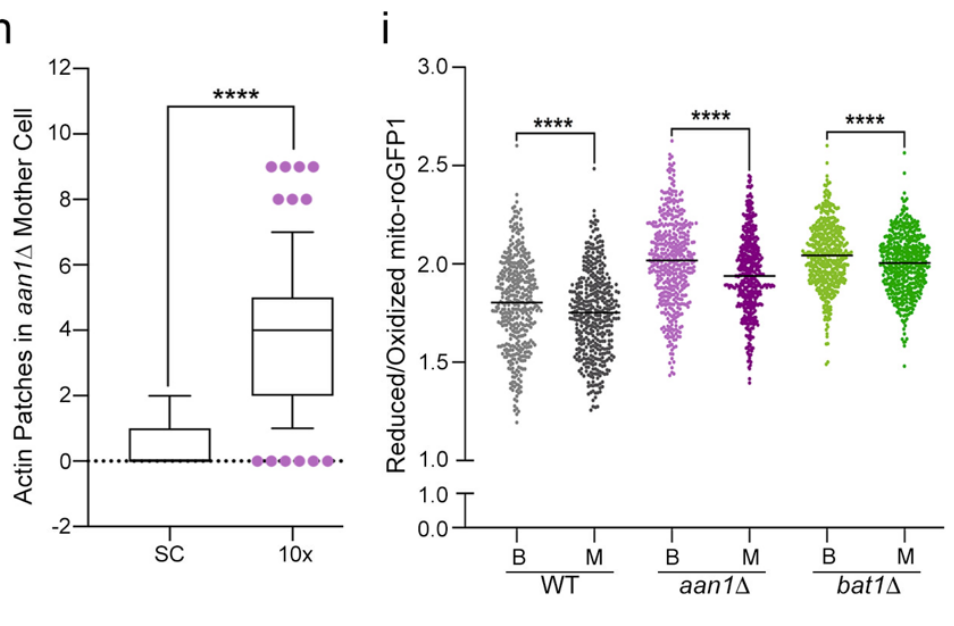

Extended Data Fig. 4: AAN1 function in modulating actin cables through effects on BCAA homeostasis.

a, Representative images of Alexa488 phalloidin-stained F-actin of mid-log phase WT, aan1 $(y \mathrm{k} / 075 c) \Delta$, bat2 $\Delta$, or aan1 $\Delta$ bat2 $\Delta$ cells. b, Quantification of actin cable abundance in WT, 
aan $1 \Delta$, bat2 $\Delta$, or aan1 $\Delta$ bat2 $\Delta$ cells. Data from representative trial. $\mathrm{n}$ of cells/strain: 78 (WT), 70

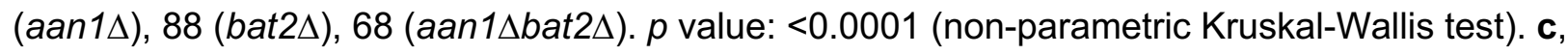
Representative images of Alexa488 phalloidin-stained F-actin of mid-log phase WT cells grown in synthetic complete (SC) medium or SC without lysine (-Lys) for $30 \mathrm{~min}$. d, Quantification of actin cable abundance in the presence or absence of lysine. Combined $\mathrm{n}$ of cells/strain/group: 129 (WT $\mathrm{SC}$ ), and 135 (WT-Lys). Data values were generated from 3 trials and analyzed using a two-tailed non-parametric Kruskal-Wallis test (n.s., not significant). e, Representative images of

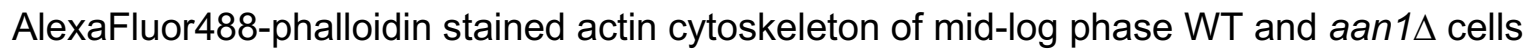
grown in synthetic complete (SC) medium or SC with leucine supplementation. Scale bar, $2 \mu \mathrm{m}$.

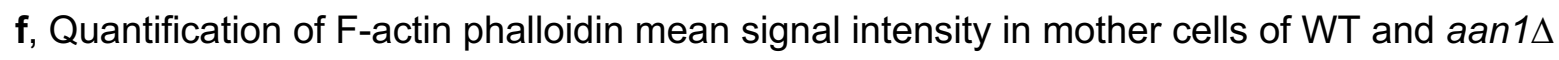
strains. Data from representative trial. $\mathrm{n}$ of cells/strain: 95 (WT in SC), 100 (WT in 10xLeu), 143

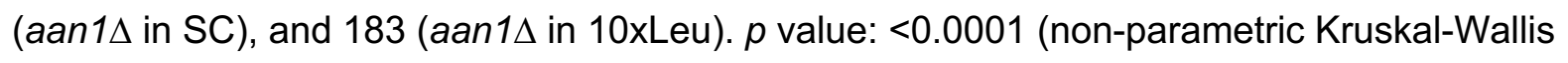
test). $\mathbf{g}$, The actin cytoskeleton phenotypes in mid-log phase aan1 $\Delta$ cells grown in SC ( $n=146$ cells) and SC with 10x leucine ( $n=114$ cells) stained with AlexaFluor488-phalloidin. Full length actin cables are $\geq 2 \mu \mathrm{m}$ and typically extend from the bud to the tip of the mother cell distal to the bud. $\mathbf{h}$, Quantification of actin patches within mother cells of aan1 $\Delta$ cells grown in SC ( $\mathrm{n}=$ 143 cells) and SC with 10x leucine ( $n=117$ cells). $p$ value: $<0.0001$ (Mann-Whitney test). i, Reduced:oxidized mito-roGFP1 ratios of mitochondria in mother and buds of mid-log phase WT, aan $1 \Delta$, and bat1 $1 \Delta$ cells. Combined $n$ of 3 trials: 419 (WT bud and mother), 461 (aan1 $1 \Delta$ bud and mother), and 486 (bat1 $\Delta$ bud and mother). $p$ value: <0.0001 (Wilcoxon matched-pair test). 


\section{Methods}

Yeast growth conditions. All S. cerevisiae strains used in this study are derivatives of the wild-

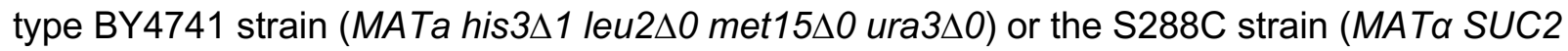
gal2 mal2 mel flo1 hap1 ho bio1 bio6) from Open Biosystems (Huntsville AL). All cultures used for imaging and RLS studies were grown to mid-log phase $\left(\mathrm{OD}_{600} 0.1-0.5\right)$ by shaking at $30^{\circ} \mathrm{C}$ in a glucose-based yeast-peptone-dextrose (YPD) or synthetic complete (SC) medium.

Yeast strain construction. All knockout strains (Supplementary Table 2) were created using homologous recombination-based methods. Genes of interest were replaced with either auxotrophic or drug resistance markers using primers and cassettes described in Supplementary Table 3. For example, yk1075c $\Delta$ cells were generated by replacing YKL075C with KanMX6, a geneticin resistant gene, using a homologous recombinant PCR fragment, which contained: flanking sequences of the $Y K L 075 C$ gene and the KanMX6 coding region from the plasmid pFA6-KanMX6 (Addgene, Watertown MA). All transformations were carried out using the lithium acetate and transformants were identified as colonies that grow on selective solid media. For example, KanMX6-positive cells were selected using YPD solid media containing $200 \mu \mathrm{g} / \mathrm{mL}$ Geneticin (Sigma-Aldrich, St. Louis MO). Selected colonies were further validated using PCR sequencing.

The same techniques were used to add fluorescent protein tags at the chromosomal loci of genes of interest using PCR fragments amplified from plasmids: pFA6a-GFP(S65ST)KanMX6 and pFA6a-GFPEnvy-KanMX6 (Addgene, Watertown MA). Selection for positive transformants was carried out as described above and confirmed by visual analysis using widefield fluorescence microscopy.

Column isolation of aged yeast cells. Cells of defined replicative age were isolated using a modification of the protocol described in Smeal et al. $1998^{31}$. Yeast strains of interest were 
grown to a mid-log phase at $30^{\circ} \mathrm{C}$ in YPD media $\left(\mathrm{OD}_{600}<0.5\right) \cdot 10^{7}$ cells were removed from the mid-log phase culture, concentrated by centrifugation using a Sorvall ST-16 tabletop centrifuge (3500 rpm for 4 min at RT), and washed twice with 1x PBS at pH 8.0. The cell pellet was resuspended with $500 \mu \mathrm{L}$ of sterile 1x PBS. To biotinylate cells, $3.0 \mathrm{mg}$ of EZ Link Sulfo-NHSLC-Biotin (Thermo Fischer Scientific, Waltham MA) was dissolved in $500 \mu \mathrm{L}$ of $1 \mathrm{x}$ PBS at room temperature (RT) and immediately added to cells. Cells were incubated for 30 min on a rotating platform at RT. Biotinylated cells were gently washed twice with 1x PBS 100 mM Glycine, inoculated into a sterile flask containing $500 \mathrm{~mL}$ of YPD media and propagated for $8 \mathrm{~h}(6-8$ generations) at $30^{\circ} \mathrm{C}$ with shaking. After propagation, cells were incubated with FluidMAGStreptavidin beads (Chemicell, Berlin Germany) at $500 \mu \mathrm{g} / 5.0 \times 10^{7}$ cells for $30 \mathrm{~min}$ at RT. Cells were then washed with YPD and applied to an LS column (Miltenyi Biotec Inc., Auburn CA) mounted in a QaudroMACS magnetic separator rack (Miltenyi Biotec Inc., Auburn CA). Labeled (old) cells were retained in the column, and unlabeled cells in the column flow-through were recovered and used as young cell controls. The column was washed with YPD until the flowthrough did not contain any cells. To isolate old cells, the LS column was removed from the magnetic separator rack and the column was washed with YPD media. Isolated of old cells were further aged by propagation as described above for $8 \mathrm{~h}$. After $8 \mathrm{~h}$, old cells were isolated as described above.

mCAD isolation of aged yeast cells. Aged-yeast cells were isolated from the miniatureChemostat Aging Device (mCAD) as described by Hendrickson et al. $2018^{32}$. Briefly, $4 \times 10^{6}$ cells were obtained from an overnight mid-log culture with a cell density of $<0.2 O D_{600}$ and washed twice with 1x PBS containing 0.25\% PEG3350. The cell pellet from washed cells was resuspended in 1x PBS, mixed with 2 mg of EZ Link Sulfo-NHS-LC-Biotin (Thermo Fischer Scientific, Waltham MA) dissolved in 1x PBS and incubate for $30 \mathrm{~min}$ at RT in a rotating 
platform mixer. Biotinylated cells were washed with 1x PBS 0.25\% PEG3350, inoculated into a sterile flask with $500 \mathrm{~mL}$ of SC media and propagated for $8 \mathrm{~h}$ at $30^{\circ} \mathrm{C}$ with shaking to mid-log growth state $\left(\mathrm{OD}_{600}<0.3\right)$. Dynabeads MyOne Streptavidin $\mathrm{C} 1$ beads (Thermo Fischer Scientific, Waltham MA) were washed twice with SC media, and resuspended in $20 \mathrm{~mL}$ of SC in a $50 \mathrm{~mL}$ conical tube. Biotinylated-labeled cells were incubated with Dynabeads $\left(9 \mu \mathrm{g} / 10^{6}\right.$ cells $)$ for 15 min at RT in rotating platform mixer. The cell mixture was transferred into a sterile glass culture tube surrounded by ring magnets, and allowed to stand and for $5 \mathrm{~min}$ at RT. Unlabeled young cells that were not immobilized in the magnetic field were removed by aspiration. Ring magnets were then removed and cells that were released were suspended in $1 \mathrm{~mL}$ of $\mathrm{SC}$ media and transferred to a Luer lock syringe for mCAD loading.

The mCAD was prepared and assembled as previously described ${ }^{32}$. Labeled cells in the Luer lock syringe were loaded onto the Luer needle entry port of the mCAD vessel. The cell loaded-vessel was placed into ring magnets for 10-15 min to immobilize the labeled cells before they were propagated in the mCAD vessel for 41-45 $\mathrm{h}$ with constant media feed using a peristaltic pump (25 mL/h) and aeration using an air pump (0.8-1.0 PSI). To collect cells, all entry ports were removed from the mCAD vessel. Unbound cells were carefully removed by a serological pipette and discarded and residual unbound cells were removed by washes with media. The vessel was removed from the magnetic rings and released cell were resuspend in SC media, washed with fresh media and resuspended in SC media.

Growth rates. Growth curves were measured using an automated plate reader (Tecan; Infinite M200, Research Triangle Park, NC) or manually using spectrophotometer (Beckman; Irvine, CA). Strain preparation for automated plate reader detection follows as previously described in Garcia et al., 2019. Each strain is grown to mid-log phase in rich, glucose-based media (YPD) and diluted to an $\mathrm{OD}_{600}$ of $0.07\left(2.0 \times 10^{6} \mathrm{cells} / \mathrm{ml}\right) .10 \mu \mathrm{l}$ of the diluted strain was added to a 
well containing $200 \mu \mathrm{I}$ YPD in a 96 -well plate. Cells were propagated at $30^{\circ} \mathrm{C}$ without shaking, and an optical density reading at $600 \mathrm{~nm}\left(\mathrm{OD}_{600}\right)$ was measured every $20 \mathrm{~min}$ for $72 \mathrm{~h}$. Alternatively, growth rates can be measured manually by a spectrophotometer. An overnight mid-log culture $\left(\mathrm{OD}_{600} 0.3-0.5\right)$ in $\mathrm{YPD}$ was prepared and diluted to $0.1 \mathrm{OD}_{600}$. Cells were propagated in a $30^{\circ} \mathrm{C}$ shaking incubator and optical density reading at $600 \mathrm{~nm}$ was measured in spectrophotometer cuvette at a 1 in 10 dilution every hour for $16 \mathrm{~h}$.

Microscopy. Wide field fluorescence imaging was carried out using an Axioskop 2 microscope equipped with a 100x/1.4 Plan-Apochromat objective (Zeiss, Thornwood NY), an Orca-ER CCD camera (Hamamatsu Corporation, Bridgewater NJ), a pE-4000 LED illumination system (coolLED, Andover UK,). The system was controlled by NIS Elements 4.60 Lambda software (Nikon). Super-resolution imaging was performed using a structured illumination microscope (NSIM S, Nikon) equipped with a 100x/1.49 oil-immersion objective lens (Nikon Instruments, Melville NY), an EMCCD Camera (iXon, Andor Technology Ltd, Belfast Ireland) and NIS Elements software (Nikon Instruments, Melville NY).

Visualization of F-actin cytoskeleton and bud scars. Mid-log phase yeast cells were concentrated to a cell density of $0.5 \mathrm{OD}_{600}$ and resuspended in $1 \mathrm{~mL}$ of growth medium for fixedcell staining. Cells were fixed in $3.7 \%$ paraformaldehyde at $30^{\circ} \mathrm{C}$ with shaking for 50 min.

We visualized the actin cytoskeleton in fixed cells by wide-field microscopy using the following protocol. Fixed-cells were washed three times with 1x PBS, followed by one wash with 1x PBT (PBS containing 1\% w/v BSA, $0.1 \%$ v/v Triton X-100, 0.1\% w/v sodium azide), and actin was stained with $2.5 \mu \mathrm{M}$ AlexaFluor488-phalloidin (Thermo Fischer Scientific, Waltham MA) for 25 min at RT in the dark. Cells were subsequently washed three times with $1 \mathrm{xPBS}$ and resuspended in 7-10 $\mu \mathrm{L}$ of SlowFade Diamond anti-fade mountant (Thermo Fischer Scientific, 
Waltham MA). $1.8 \mu \mathrm{L}$ of stained cells prepped in mountant solution were placed on a glass slide and covered with a \#1.5 coverslip. AlexaFluor488-phalloidin was excited with a $470 \mathrm{~nm}$ LED and an ET470/40x filter (Chroma, Bellow Falls VT). Z-stacks were captured at $0.3 \mu \mathrm{m}$ intervals using 1x1 binning, 200-300 ms exposure time, and gain of 116. Using Volocity 5.5 (Quorum Technologies Ltd, Lewes UK), images were deconvolved using a constrained iterative restoration algorithm assuming $507 \mathrm{~nm}$ emission wavelength with a $100 \%$ confidence limit and 60 iterations. All images were contrast-enhanced with similar parameters.

Cells visualized by SIM were fixed by paraformaldehyde as previously described. Bud scars were stained first with $5 \mu \mathrm{g} / \mathrm{mL}$ AlexaFluor594-WGA (Thermo Fischer Scientific, Waltham MA) and incubated at RT in the dark for $30 \mathrm{~min}$. Cells were washed twice with 1x PBS and once with 1x PBT. After bud scar staining, the actin cytoskeleton was stained with $2.5 \mu \mathrm{M}$ AlexaFluor488-phalloidin staining as previously described. Additional preparations were required for SIM imaging and used the following protocol described in Higuchi-Sanabria et al $2016^{72}$. Briefly, stained cells adhered to coverslips coated with concanavalin-A were mounted on a slide with ProLong Diamond anti-fade mountant (Thermo Fischer Scientific, Waltham MA). Prepared slides cured overnight in the dark at RT. Used N-SIM S instrument as described above with a camera EM gain of 200. AlexaFluor488-phalloidin was excited with a $488 \mathrm{~nm}$ laser at $45 \%$ power with $300 \mathrm{~ms}$ exposure time and AlexaFluor594-WGA was excited with a $561 \mathrm{~nm}$ laser at $10 \%$ power with 200 ms exposure time. A z-series of $0.125 \mu \mathrm{m}$ intervals with $1 \times 1$ binning was collected through the entire cell. SIM images were reconstructed using parameters described in Higuchi-Sanabria et $\mathrm{al}^{72}$. All images were contrast-enhanced with similar parameters.

Immunofluorescence staining. Mid-log phase yeast cells $\left(0.2-0.5 \mathrm{OD}_{600}\right.$ cell density) were fixed with $3.7 \%$ paraformaldehyde for $1 \mathrm{~h}$ at $30^{\circ} \mathrm{C}$ with shaking. Immunofluorescence staining was performed as described in detail by Higuchi-Sanabria et al. $2016^{72}$. Fixed cells were treated 
with $50 \mu \mathrm{g} / \mathrm{mL}$ of Zymolyase 20T (Seikagaku Inc., Tokyo Japan) for $30 \mathrm{~min}$ to remove the yeast cell wall. Spheroplasts $(10 \mu \mathrm{L})$ were resuspended in $100 \mu \mathrm{L} 1 \times$ PBS and placed onto a polylysine-coated 22x22 mm coverslip, and incubated in a dark humid chamber for $35 \mathrm{~min}$ at RT. Unbound spheroplasts were gently washed away with 1x PBT, and the spheroplast-coated coverslip was placed onto $30 \mu \mathrm{L}$ of $1: 100$ diluted primary $13 x M y c$ antibody and incubated for $2 \mathrm{~h}$ in a dark humid chamber at RT. Coverslip was rinsed four times with 1x PBT and incubated in $30 \mu \mathrm{L}$ of 1:100 diluted secondary antibody (Alexa Fluor plus 488, Thermo Fischer Scientific, Waltham MA) for $1 \mathrm{~h}$. Coverslip was rinsed with $1 \mathrm{x}$ PBT and mounted on a slide with $1.5 \mu \mathrm{L}$ of SlowFade mountant (Thermo Fischer Scientific, Waltham MA). The coverslip edges were sealed with clear nail polish and slides were stored at $4^{\circ} \mathrm{C}$.

Actin cable stability and abundance analysis. For cell growth curves, yeast cells were incubated with $8 \mu \mathrm{M}$ Latrunculin-A (Lat-A, Sigma-Aldrich, St. Louis MO) for $3 \mathrm{~d}$, taking cell density measurements $\left(\mathrm{OD}_{600}\right)$ every hour. Yeast cells were treated with 1-2 $\mu \mathrm{M}$ Lat-A for 20 min and 5 min interval samplings were acquired, fixed, and stained to determine actin cable stability. Stained cells were imaged with wide-field microscopy as described above. Quantified deconvolved wide-field images for the presence or absence of actin cables in mother cells of both DMSO and Lat-A treated yeast cells. The binary quantification determined the rate of actin cable loss during the Lat-A time-course.

Actin cable abundance was quantified using deconvolved wide-field images. Actin cables were mapped from the bud neck to the mother cell tip using the Volocity tracing tool and the number of actin cable tracings determined the actin cable abundance.

Analysis of Mitochondrial Redox State using mito-roGFP1. A ratiometric probe, mito-GFP1, was used to measure the redox state of the mitochondrion. We performed imaging and analysis as previously described in McFaline-Figueroa et al. 2011 and Vevea et al. $2013^{19,37}$. Ratiometric 
probe, mito-roGFP1, was integrated at the $H O$ locus. Acquired images on a wide-field microscope with reduced and oxidized states excited at $470 \mathrm{~nm}$ and $365 \mathrm{~nm}$ with a GFP filter cube without an excitation filter (Zeiss filter 46HE, dichroic FT 515, emission $535 \mathrm{~nm} / 30$ ). Images were captured images with a z-series at $0.3 \mu \mathrm{m}$ intervals through the entire depth of the cell. The imaging deconvolution parameters were 60 iterations of a constrained iterative restoration algorithm with a calculated PSF using the following parameters: $\lambda_{\text {ex }} 507 \mathrm{~nm}$ and 100\% confidence limit (Volocity 5.5, Quorum Technologies Ltd, Lewes UK). Once the background was subtracted and thresholded each image, the reduced/oxidized ratio calculated by dividing the intensity of the reduced channel $\left(\lambda_{\mathrm{ex}}=470 \mathrm{~nm}, \lambda_{\mathrm{em}}=525 \mathrm{~nm}\right)$ over the intensity in the oxidized channel $\left(\lambda_{\mathrm{ex}}=365 \mathrm{~nm}, \lambda_{\mathrm{em}}=525 \mathrm{~nm}\right)$, in Volocity software.

RLS analysis. RLS measurements were performed as previously described, without alphafactor synchronization ${ }^{30}$. Selected strains stored at $-80^{\circ} \mathrm{C}$ were streaked onto YPD plates and grown for 2 days at $30^{\circ} \mathrm{C}$. A swab from a colony patch was grown overnight in liquid YPD at $30^{\circ} \mathrm{C}$ to mid-log phase. $10 \mu \mathrm{L}$ of cell suspension was streaked onto a YPD plate. Small-budded cells were isolated and arranged in a matrix using a micromanipulator mounted on a dissecting microscope (Sporeplay, Singer Instruments, Somerset UK). Upon a complete budding event, mother cells were removed and discarded to leave only virgin cells. The time and number of subsequent daughter cells produced by each virgin mother cell were recorded until all replication ceased.

Branched-chain amino acid levels. Cell lysate was prepared from 8-10 OD 600 of mid-log phase cells by vortexing cells with $0.5 \mathrm{~mm}$ glass beads in chilled $1 \mathrm{x}$ PBS for $5 \mathrm{~min}$. Beads and debris were pelleted from the cell suspension at 13 RPM for $0.5 \mathrm{~s}$ and the supernatant was placed on ice. Total protein concentration was measured using a Pierce BCA protein assay kit 
(Thermo Fischer Scientific, Waltham MA). BCAA levels were determined using the Branched Chain Amino Acid Assay kit (Cell Biolabs Inc., San Diego CA) following the manufacturer's instructions. BCAA levels were measured in triple technical replicates against negative control samples lacking leucine dehydrogenase. Levels of free BCAAs extracted were normalized to the extracted protein concentration from cell lysates.

RNA Sequencing. The transcriptome was analyzed as previously described in Liao et al. $2020^{73}$. RNA was extracted from mid-log phase WT and $y k 1075 c \Delta$ cells using the RNeasy kit (Qiagen, Germantown, MD). RNA quality was analyzed with Agilent 2100 Bioanalyzer using a Plant RNA Nano chip and RNA Integrity Number (RIN) scores higher than 9 were sequenced. RNA library preparations and sequencing reactions were conducted at GENEWIZ, LLC. RNA sequencing libraries were prepared using the NEB Next Ultra RNA Library Prep Kit for Illumina using manufacturer's instructions (NEB, Ipswich MA). The sequencing library was validated on the Agilent Tape Station (Agilent Technologies, Santa Clara CA), and quantified using a Qubit 2.0 Fluorometer (Invitrogen, Thermo Fischer Scientific, Waltham MA) as well as by quantitative PCR (KAPA Biosystems, Sigma-aldrich, St. Louis MA). The sequencing libraries were clustered on a single lane of a flowcell. After clustering, the flowcell was loaded on the Illumina HiSeq instrument 4000 according to the manufacturer's instructions. The samples were sequenced using a 2x150 bp Paired End (PE) configuration. Sequence reads were trimmed to remove possible adaptor sequences and nucleotides with poor quality using Trimmomatic v.0.36. The trimmed reads were mapped to the Saccharomyces cerevisiae S288C reference genome available on ENSEMBL using the STAR aligner v.2.5.2b. Unique gene hit counts were calculated by using feature counts from the Subread package v.1.5.2. After extraction of gene hit counts, the gene hit counts table was used for downstream differential expression analysis. Using DESeq2, a comparison of gene expression between WT and yk1075c $\Delta$ cells was performed. The Wald test was used to generate $p$-values and $\log _{2}$ fold changes. Genes with a 
p-value $<0.05$ and absolute $\log _{2}$ fold change $>1$ were called as differentially expressed genes. Yeastract+ database was to determine GO terms from RNA seq ${ }^{74}$.

cDNA synthesis and quantitative PCR. RNA was extracted from cultured mid-log phase WT or $y$ k1075c $\Delta$ cells using the RNeasy kit (QIAGEN, Germantown, MD). Genomic DNA contamination was removed using TURBO DNA-free Kit (Ambion, Thermo Fischer Scientific, Waltham MA). $1 \mathrm{mg}$ of DNA-free RNA was used for cDNA synthesis with SuperScript IV FirstStrand Synthesis System (Invitrogen, Thermo Fischer Scientific, Waltham MA). cDNA was diluted and used for quantitative PCR reaction with PowerUp SYBR Green Master Mix (Applied Biosystems, Foster City CA). Primers for qPCR were designed using NCBI Primer Blast (https://www.ncbi.nlm.nih.gov/tools/primer-blast/) with a PCR product size of $100 \mathrm{bp}$ and max Tm difference of $2^{\circ} \mathrm{C}$. For each specified gene, $\Delta \mathrm{CT}$ was calculated as $\mathrm{CT}_{\text {gene }}-\mathrm{CT}_{\text {actin }}$, and fold change was calculated as $2^{-\triangle \Delta C T}$ with actin serving as the endogenous control for each sample.

\section{Quantification and Statistical Analysis. GraphPad Prism 9 (GraphPad Software, San Diego} CA) was used to conduct all statistical analyses and create all graphs. All tests applied twotailed statistical analysis. $p$ values for simple two-group comparison were determined with a twotailed Student's t-test for parametric distributions and a Mann-Whitney test for non-parametric data. For multiple group comparisons, $p$ values were determined by a 1-way ANOVA KruskalWallis test with Dunn's post hoc test for non-parametric distributions. Survival curves were statistically compared using a Log-rank (Mantel-Cox) test. Bar graphs show the mean and SEM; in box and whisker graphs, the box represents the middle quartile, the midline represents the median, whiskers show the $10^{\text {th }}$ and $90^{\text {th }}$ percentiles, and points indicate outliers. For all tests, $p$ values are classified as follows: ${ }^{*} p<0.05 ;{ }^{* *} p<0.01 ;{ }^{* * *} p<0.001 ;{ }^{* * * *} p<0.0001$, unless exact $p$ values are noted in the figure legends. 
bioRxiv preprint doi: https://doi.org/10.1101/2021.10.07.463535; this version posted October 7, 2021. The copyright holder for this preprint (which was not certified by peer review) is the author/funder, who has granted bioRxiv a license to display the preprint in perpetuity. It is made available under aCC-BY-NC-ND 4.0 International license. 


\section{References}

1. Li, Z. et al. Aging-Impaired Filamentous Actin Polymerization Signaling Reduces Alveolar Macrophage Phagocytosis of Bacteria. J. Immunol. 199, 3176-3186 (2017).

2. Noble, J. M., Ford, G. A. \& Thomas, T. H. Effect of aging on CD11b and CD69 surface expression by vesicular insertion in human polymorphonuclear leucocytes. Clin. Sci. 97, 323-329 (1999).

3. Piazzolla, G., Tortorella, C., Serrone, M., Jirillo, E. \& Antonaci, S. Modulation of cytoskeleton assembly capacity and oxidative response in aged neutrophils. Immunopharmacol Immunotoxicol 20, 251-266 (1998).

4. Plackett, T. P., Boehmer, E. D., Faunce, D. E. \& Kovacs, E. J. Aging and innate immune cells. J. Leukoc. Biol. 76, 291-299 (2004).

5. Rao, K. M. Age-related decline in ligand-induced actin polymerization in human leukocytes and platelets. J Gerontol 41, 561-566 (1986).

6. Rao, K. M., Currie, M. S., Padmanabhan, J. \& Cohen, H. J. Age-related alterations in actin cytoskeleton and receptor expression in human leukocytes. J Gerontol 47, B37-44 (1992).

7. Brock, M. A. \& Chrest, F. Differential regulation of actin polymerization following activation of resting T lymphocytes from young and aged mice. J. Cell Physiol. 157, 367-378 (1993).

8. Garcia, G. G. \& Miller, R. A. Age-dependent defects in TCR-triggered cytoskeletal rearrangement in CD4+ T cells. J. Immunol. 169, 5021-5027 (2002).

9. Garcia, G. G. \& Miller, R. A. Age-related defects in the cytoskeleton signaling pathways of CD4 T cells. Ageing Res Rev 10, 26-34 (2011).

10. Tamir, A., Eisenbraun, M. D., Garcia, G. G. \& Miller, R. A. Age-dependent alterations in the assembly of signal transduction complexes at the site of T cell/APC interaction. J. Immunol. $165,1243-1251(2000)$.

11. Kasper, G. et al. Insights into mesenchymal stem cell aging: involvement of antioxidant defense and actin cytoskeleton. Stem Cells 27, 1288-1297 (2009). 
12. Li, X. \& Larsson, L. Maximum shortening velocity and myosin isoforms in single muscle fibers from young and old rats. Am. J. Physiol. 270, C352-60 (1996).

13. Larsson, L., Li, X. \& Frontera, W. R. Effects of aging on shortening velocity and myosin isoform composition in single human skeletal muscle cells. Am. J. Physiol. 272, C638-49 (1997).

14. Degens, H., Yu, F., Li, X. \& Larsson, L. Effects of age and gender on shortening velocity and myosin isoforms in single rat muscle fibres. Acta Physiol Scand 163, 33-40 (1998).

15. Yu, F., Degens, H., Li, X. \& Larsson, L. Gender- and age-related differences in the regulatory influence of thyroid hormone on the contractility and myosin composition of single rat soleus muscle fibres. Pflugers Arch. 437, 21-30 (1998).

16. Höök, P., Sriramoju, V. \& Larsson, L. Effects of aging on actin sliding speed on myosin from single skeletal muscle cells of mice, rats, and humans. Am. J. Physiol. Cell Physiol. 280, C782-8 (2001).

17. Aguilaniu, H., Gustafsson, L., Rigoulet, M. \& Nyström, T. Asymmetric inheritance of oxidatively damaged proteins during cytokinesis. Science 299, 1751-1753 (2003).

18. Liu, B. et al. The polarisome is required for segregation and retrograde transport of protein aggregates. Cell 140, 257-267 (2010).

19. McFaline-Figueroa, J. R. et al. Mitochondrial quality control during inheritance is associated with lifespan and mother-daughter age asymmetry in budding yeast. Aging Cell 10, 885895 (2011).

20. Higuchi, R. et al. Actin dynamics affect mitochondrial quality control and aging in budding yeast. Curr. Biol. 23, 2417-2422 (2013).

21. Pernice, W. M., Vevea, J. D. \& Pon, L. A. A role for Mfb1p in region-specific anchorage of high-functioning mitochondria and lifespan in Saccharomyces cerevisiae. Nat. Commun. 7, 10595 (2016).

22. Hughes, A. L. \& Gottschling, D. E. An early age increase in vacuolar $\mathrm{pH}$ limits mitochondrial 
function and lifespan in yeast. Nature 492, 261-265 (2012).

23. Katajisto, P. et al. Stem cells. Asymmetric apportioning of aged mitochondria between daughter cells is required for stemness. Science 348, 340-343 (2015).

24. Yang, H.-C. \& Pon, L. A. Actin cable dynamics in budding yeast. Proc. Natl. Acad. Sci. USA 99, 751-756 (2002).

25. Okada, K., Ravi, H., Smith, E. M. \& Goode, B. L. Aip1 and cofilin promote rapid turnover of yeast actin patches and cables: a coordinated mechanism for severing and capping filaments. Mol. Biol. Cell 17, 2855-2868 (2006).

26. Buttery, S. M., Yoshida, S. \& Pellman, D. Yeast formins Bni1 and Bnr1 utilize different modes of cortical interaction during the assembly of actin cables. Mol. Biol. Cell 18, 18261838 (2007).

27. Yu, J. H., Crevenna, A. H., Bettenbühl, M., Freisinger, T. \& Wedlich-Söldner, R. Cortical actin dynamics driven by formins and myosin V. J. Cell Sci. 124, 1533-1541 (2011).

28. Adams, A. E. \& Pringle, J. R. Relationship of actin and tubulin distribution to bud growth in wild-type and morphogenetic-mutant Saccharomyces cerevisiae. J. Cell Biol. 98, 934-945 (1984).

29. Pruyne, D., Legesse-Miller, A., Gao, L., Dong, Y. \& Bretscher, A. Mechanisms of polarized growth and organelle segregation in yeast. Annu. Rev. Cell Dev. Biol. 20, 559-591 (2004).

30. Erjavec, N., Cvijovic, M., Klipp, E. \& Nyström, T. Selective benefits of damage partitioning in unicellular systems and its effects on aging. Proc. Natl. Acad. Sci. USA 105, 1876418769 (2008).

31. Smeal, T., Claus, J., Kennedy, B., Cole, F. \& Guarente, L. Loss of transcriptional silencing causes sterility in old mother cells of S. cerevisiae. Cell 84, 633-642 (1996).

32. Hendrickson, D. G. et al. A new experimental platform facilitates assessment of the transcriptional and chromatin landscapes of aging yeast. Elife 7, (2018).

33. Fujiwara, I., Zweifel, M. E., Courtemanche, N. \& Pollard, T. D. Latrunculin A accelerates 
actin filament depolymerization in addition to sequestering actin monomers. Curr. Biol. 28, 3183-3192.e2 (2018).

34. Ayscough, K. R. et al. High rates of actin filament turnover in budding yeast and roles for actin in establishment and maintenance of cell polarity revealed using the actin inhibitor latrunculin-A. J. Cell Biol. 137, 399-416 (1997).

35. Teng, X. et al. Genome-wide consequences of deleting any single gene. Mol. Cell 52, 485494 (2013).

36. Hanson, G. T. et al. Investigating mitochondrial redox potential with redox-sensitive green fluorescent protein indicators. J. Biol. Chem. 279, 13044-13053 (2004).

37. Vevea, J. D., Wolken, D. M. A., Swayne, T. C., White, A. B. \& Pon, L. A. Ratiometric biosensors that measure mitochondrial redox state and ATP in living yeast cells. J. Vis. Exp. (2013). doi:10.3791/50633

38. Breker, M., Gymrek, M. \& Schuldiner, M. A novel single-cell screening platform reveals proteome plasticity during yeast stress responses. J. Cell Biol. 200, 839-850 (2013).

39. Kohlhaw, G. B. Leucine biosynthesis in fungi: entering metabolism through the back door. Microbiol. Mol. Biol. Rev. 67, 1-15, table of contents (2003).

40. Colón, M. et al. Saccharomyces cerevisiae Bat1 and Bat2 aminotransferases have functionally diverged from the ancestral-like Kluyveromyces lactis orthologous enzyme. PLoS One 6, e16099 (2011).

41. González, J. et al. Diversification of Transcriptional Regulation Determines Subfunctionalization of Paralogous Branched Chain Aminotransferases in the Yeast Saccharomyces cerevisiae. Genetics 207, 975-991 (2017).

42. Heitman, J., Movva, N. R. \& Hall, M. N. Targets for cell cycle arrest by the immunosuppressant rapamycin in yeast. Science 253, 905-909 (1991).

43. Chou, M. M. \& Blenis, J. The $70 \mathrm{kDa}$ S6 kinase: regulation of a kinase with multiple roles in mitogenic signalling. Curr. Opin. Cell Biol. 7, 806-814 (1995). 
44. Kingsbury, J. M., Sen, N. D. \& Cardenas, M. E. Branched-Chain Aminotransferases Control TORC1 Signaling in Saccharomyces cerevisiae. PLoS Genet. 11, e1005714 (2015).

45. Loewith, R. et al. Two TOR complexes, only one of which is rapamycin sensitive, have distinct roles in cell growth control. Mol. Cell 10, 457-468 (2002).

46. Giaever, G. et al. Functional profiling of the Saccharomyces cerevisiae genome. Nature 418, 387-391 (2002).

47. Barbet, N. C. et al. TOR controls translation initiation and early G1 progression in yeast. Mol. Biol. Cell 7, 25-42 (1996).

48. Reinke, A. et al. TOR complex 1 includes a novel component, Tco89p (YPL180w), and cooperates with Ssd1p to maintain cellular integrity in Saccharomyces cerevisiae. J. Biol. Chem. 279, 14752-14762 (2004).

49. Kunz, J. et al. Target of rapamycin in yeast, TOR2, is an essential phosphatidylinositol kinase homolog required for G1 progression. Cell 73, 585-596 (1993).

50. González, A. et al. TORC1 promotes phosphorylation of ribosomal protein S6 via the AGC kinase Ypk3 in Saccharomyces cerevisiae. PLoS One 10, e0120250 (2015).

51. Powers, T. \& Walter, P. Regulation of ribosome biogenesis by the rapamycin-sensitive TOR-signaling pathway in Saccharomyces cerevisiae. Mol. Biol. Cell 10, 987-1000 (1999).

52. Cardenas, M. E., Cutler, N. S., Lorenz, M. C., Di Como, C. J. \& Heitman, J. The TOR signaling cascade regulates gene expression in response to nutrients. Genes Dev. 13, 3271-3279 (1999).

53. Brauer, M. J. et al. Coordination of growth rate, cell cycle, stress response, and metabolic activity in yeast. Mol. Biol. Cell 19, 352-367 (2008).

54. Kim, E., Goraksha-Hicks, P., Li, L., Neufeld, T. P. \& Guan, K.-L. Regulation of TORC1 by Rag GTPases in nutrient response. Nat. Cell Biol. 10, 935-945 (2008).

55. Binda, M. et al. The Vam6 GEF controls TORC1 by activating the EGO complex. Mol. Cell 35, 563-573 (2009). 
56. Neklesa, T. K. \& Davis, R. W. A genome-wide screen for regulators of TORC1 in response to amino acid starvation reveals a conserved Npr2/3 complex. PLoS Genet. 5, e1000515 (2009).

57. Panchaud, N., Péli-Gulli, M.-P. \& De Virgilio, C. Amino acid deprivation inhibits TORC1 through a GTPase-activating protein complex for the Rag family GTPase Gtr1. Sci. Signal. 6, ra42 (2013).

58. Moseley, J. B. \& Goode, B. L. The yeast actin cytoskeleton: from cellular function to biochemical mechanism. Microbiol. Mol. Biol. Rev. 70, 605-645 (2006).

59. Mishra, M., Huang, J. \& Balasubramanian, M. K. The yeast actin cytoskeleton. FEMS Microbiol. Rev. 38, 213-227 (2014).

60. Higuchi-Sanabria, R. et al. Spatial regulation of the actin cytoskeleton by HSF-1 during aging. Mol. Biol. Cell 29, 2522-2527 (2018).

61. Baird, N. A. et al. HSF-1-mediated cytoskeletal integrity determines thermotolerance and life span. Science 346, 360-363 (2014).

62. Weisman, L. S. Organelles on the move: insights from yeast vacuole inheritance. Nat. Rev. Mol. Cell Biol. 7, 243-252 (2006).

63. Gourlay, C. W., Carpp, L. N., Timpson, P., Winder, S. J. \& Ayscough, K. R. A role for the actin cytoskeleton in cell death and aging in yeast. J. Cell Biol. 164, 803-809 (2004).

64. Funchal, C. et al. Morphological alterations and cell death provoked by the branched-chain alpha-amino acids accumulating in maple syrup urine disease in astrocytes from rat cerebral cortex. Cell Mol. Neurobiol. 25, 851-867 (2005).

65. Aris, J. P. et al. Autophagy and leucine promote chronological longevity and respiration proficiency during calorie restriction in yeast. Exp. Gerontol. 48, 1107-1119 (2013).

66. Maruyama, Y., Ito, T., Kodama, H. \& Matsuura, A. Availability of Amino Acids Extends Chronological Lifespan by Suppressing Hyper-Acidification of the Environment in Saccharomyces cerevisiae. PLoS One 11, e0151894 (2016). 
67. Hinnebusch, A. G. Translational regulation of GCN4 and the general amino acid control of yeast. Annu. Rev. Microbiol. 59, 407-450 (2005).

68. Owen, C. H., DeRosier, D. J. \& Condeelis, J. Actin crosslinking protein EF-1a of Dictyostelium discoideum has a unique bonding rule that allows square-packed bundles. $J$. Struct. Biol. 109, 248-254 (1992).

69. Gross, S. R. \& Kinzy, T. G. Improper organization of the actin cytoskeleton affects protein synthesis at initiation. Mol. Cell. Biol. 27, 1974-1989 (2007).

70. Visweswaraiah, J. et al. Evidence that eukaryotic translation elongation factor $1 \mathrm{~A}(\mathrm{eEF} 1 \mathrm{~A})$ binds the Gcn2 protein C terminus and inhibits Gcn2 activity. J. Biol. Chem. 286, 3656836579 (2011).

71. Perez, W. B. \& Kinzy, T. G. Translation elongation factor 1 A mutants with altered actin bundling activity show reduced aminoacyl-tRNA binding and alter initiation via elF2 $\alpha$ phosphorylation. J. Biol. Chem. 289, 20928-20938 (2014).

72. Higuchi-Sanabria, R., Swayne, T. C., Boldogh, I. R. \& Pon, L. A. Imaging of the actin cytoskeleton and mitochondria in fixed budding yeast cells. Methods Mol. Biol. 1365, 63-81 (2016).

73. Liao, P.-C., Wolken, D. M. A., Serrano, E., Srivastava, P. \& Pon, L. A. MitochondriaAssociated Degradation Pathway (MAD) Function beyond the Outer Membrane. Cell Rep. 32, 107902 (2020).

74. Monteiro, P. T. et al. YEASTRACT+: a portal for cross-species comparative genomics of transcription regulation in yeasts. Nucleic Acids Res. 48, D642-D649 (2020).

\section{Acknowledgments}

We thank the members of the Pon laboratory for their technical assistances and valuable discussion. This work was supported by grants from the National Institutes of Health $(\mathrm{NIH})$ (GM122589 and AG051047) to LP, (AG055326 and DK007647) to CNS, (AR070013) to EJG 
and (AG65200 and DK007647) to RH-S. N-SIM microscope used in this study are supported in part through the $\mathrm{NIH} / \mathrm{NCl}$ grant (CA013696 and OD014584).

\section{Data Availability}

RNA seq data was deposited in a publicly available repository with the accession code: GSE174157.

\section{Author Contributions}

This study was built on the findings from screens generated by TGL and TMH. RH-S initially conceived and conducted initial experiments for this project. CNS further conceived this project, conducted experiments with major contributions from EJG, CAT and EJY, and wrote the manuscript. LAP conceived, directed the project, and wrote the manuscript. All authors discussed and interpreted the data together.

\section{Competing Interests}

All authors declare no competing financial interests. 